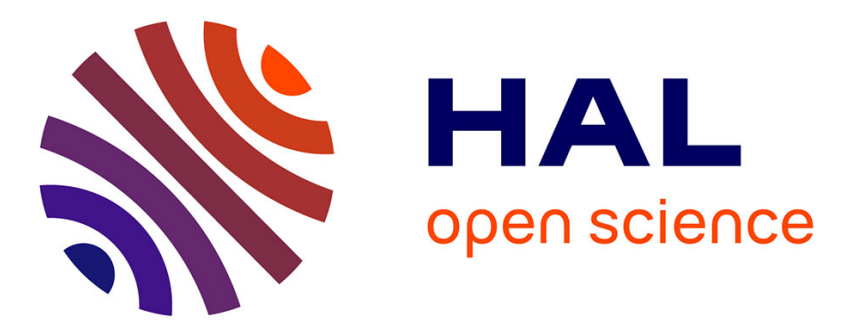

\title{
Investigation of the competition between void coalescence and macroscopic strain localization using the periodic homogenization multiscale scheme
}

\author{
Jianchang Zhu, Mohamed Ben Bettaieb, Farid Abed-Meraim
}

\section{- To cite this version:}

Jianchang Zhu, Mohamed Ben Bettaieb, Farid Abed-Meraim. Investigation of the competition between void coalescence and macroscopic strain localization using the periodic homogenization multiscale scheme. Journal of the Mechanics and Physics of Solids, 2020, 143, pp.104042. 10.1016/j.jmps.2020.104042 . hal-02901420

\section{HAL Id: hal-02901420 \\ https://hal.science/hal-02901420}

Submitted on 17 Jul 2020

HAL is a multi-disciplinary open access archive for the deposit and dissemination of scientific research documents, whether they are published or not. The documents may come from teaching and research institutions in France or abroad, or from public or private research centers.
L'archive ouverte pluridisciplinaire HAL, est destinée au dépôt et à la diffusion de documents scientifiques de niveau recherche, publiés ou non, émanant des établissements d'enseignement et de recherche français ou étrangers, des laboratoires publics ou privés. 


\title{
Investigation of the competition between void coalescence and macroscopic strain localization using the periodic homogenization multiscale scheme
}

\author{
J.C. Zhu' ${ }^{1}$ M. Ben Bettaieb ${ }^{1,2 *}$, F. Abed-Meraim ${ }^{1,2}$ \\ ${ }^{1}$ Arts et Metiers Institute of Technology, CNRS, Université de Lorraine, LEM3, F-57000, Metz, France \\ ${ }^{2}$ DAMAS, Laboratory of Excellence on Design of Alloy Metals for low-mAss Structures, Université de \\ Lorraine, France
}

\begin{abstract}
In most voided metallic materials, the failure process is often driven by the competition between the phenomena of void coalescence and plastic strain localization. This paper proposes a new numerical approach that allows an accurate description of such a competition. Within this strategy, the ductile solid is assumed to be made of an arrangement of periodic voided unit cells. Each unit cell, assumed to be representative of the voided material, may be regarded as a heterogeneous medium composed of two main phases: a central primary void surrounded by a metal matrix, which can itself be assumed to be voided. The mechanical behavior of the unit cell is then modeled by the periodic homogenization multiscale scheme. To predict the occurrence of void coalescence and macroscopic strain localization, the above multiscale scheme is coupled with several relevant criteria and indicators (among which the bifurcation approach and an energy-based coalescence criterion). The proposed approach is used for examining the occurrence of failure under two loading configurations: loadings under proportional stressing (classically used in unit cell computations to study the effect of stress state on void growth and coalescence), and loadings under proportional in-plane strain paths (traditionally used for predicting forming limit diagrams). It turns out from these numerical investigations that macroscopic strain localization acts as precursor to void coalescence when the unit cell is proportionally stressed. However, for loadings under proportional in-plane strain paths, only macroscopic strain localization may occur, while void coalescence is not possible. Meanwhile, the relations between the two configurations of loading are carefully explained within these two failure mechanisms. An interesting feature of the proposed numerical strategy is that it is flexible enough to be applied for a wide range of void shapes, void distributions, and matrix
\end{abstract}

\footnotetext{
*Email address of the corresponding author: Mohamed.BenBettaieb@ensam.eu (M. Ben Bettaieb)
} 
mechanical behavior. To illustrate the broad applicability potential of the approach, the effect of secondary voids on the occurrence of macroscopic strain localization is investigated. The results of this analysis reveal that the presence of secondary voids promotes the occurrence of macroscopic strain localization, especially for positive strain-path ratios.

Keywords: Voided materials; Periodic homogenization; Unit cell computation; Strain localization; Void coalescence; Stress triaxiality; In-plane strain paths.

\section{Introduction}

The ductility of thin metal sheets is often limited by the onset of ductile failure. Therefore, this phenomenon is central in structural integrity assessment together with corrosion and fatigue. Several possible failure scenarios may occur during plastic forming operations. In this field, one can quote at least three main scenarios. The first one takes place only for very pure metals. In this case, material fails without damage occurrence, owing to the absence of void nucleation sites. In such circumstances, the deformation state is homogeneous at the beginning of the loading, and the deformation starts concentrating in narrow bands at a certain limit strain. The initiation of such bands marks the development of localized necking in the material. The second scenario corresponds to the localization of plastic strain into narrow bands due to various possible softening mechanisms. Ultimately, following the accumulation of large plastic strains and the increase of stress triaxiality in the necked regions, voids nucleate, grow and coalesce to lead to final material failure. The third mechanism involves damage initiation within the material prior to plastic strain localization. The softening induced by the accumulated porosity is sufficient to counteract the strain hardening capacity of the material, which leads to plastic strain localization in narrow bands. An exhaustive analysis of the different failure mechanisms and the competition between them has been reported in Tekoğlu et al. (2015). It is now well known that the initiation of ductile failure and the competition between void coalescence and plastic strain localization are sensitively dependent on the stress state applied to the metal sheets. To thoroughly analyze these fundamental aspects, various experiments have been designed in several pioneering contributions. In this area, one can quote Bao and Wierzbicki (2004), who have experimentally highlighted that void growth is the dominant failure mode for high stress triaxiality, while failure for low stress triaxiality is mainly governed by the combination of shear and void growth modes. These observations have been confirmed by Barsoum and Faleskog (2007), who have experimentally established that the onset of ductile failure is additionally dependent on the Lode parameter $L$, and not only on the stress triaxiality ratio $T$, especially for low values of $T$. The combined effect of stress triaxiality ratio and Lode parameter on the failure behavior has also been confirmed by the experimental program conducted by Driemeier et al. (2010). Although some observations have been ascertained by quantitative experimental testing, the comprehensive information about the underlying mechanisms, such as 
void growth, detection of localization in the specimens, and onset of void coalescence is still difficult to reach. To overcome this difficulty, profound knowledge on ductile failure in voided materials can mainly be acquired through theoretical approaches. These theoretical approaches can be classified into two main families: micromechanical models and numerical approaches based on unit cell computations.

The class of micromechanical models has been initiated by the pioneering work of Gurson (1977), who has derived, on the basis of limit-analysis theory, a plastic potential describing the plastic flow of a representative volume element defined by a spherical void embedded in a rigid perfectly plastic matrix. The original Gurson model is based on several restrictive assumptions such as: only the effect of void growth on the mechanical behavior is considered, the voids are initially spherical and remain spherical during the growth process, and the metal matrix is dense. These restrictive assumptions limit the Gurson model capability of providing accurate predictions of the mechanical behavior. Consequently, the original Gurson model has been largely extended in the literature. The most widely-known extension has been developed in Tvergaard and Needleman (1984) to consider the effect of nucleation of new voids and coalescence of existing voids on the mechanical behavior. In this extension, referred to as the GTN model, the final material failure has been predicted by using an empirical coalescence criterion. The numerical predictions based on the GTN model have been favorably compared with various experimental results (Tvergaard and Needleman, 1984). To analyze the competition between void coalescence and strain localization, the GTN model has been coupled in Mansouri et al. (2014) and Chalal et al. (2015) with the Rice bifurcation theory (Rudnicki and Rice, 1975; Rice, 1976). This theory is based on the loss of ellipticity of the governing equations. Hence, to predict strain localization via the Rice bifurcation theory, the expression of the analytical tangent modulus needs to be derived from the constitutive equations. Despite their well-recognized interest, the extended versions of the Gurson model present some limitations and drawbacks in the analysis of the different metal failure scenarios (e.g., by void coalescence or strain localization). In fact, these models are generally based on heuristic extensions of the original Gurson model without sound physical foundations. Furthermore, they involve additional material parameters to better reproduce the experimental results (such as parameters $q_{1}, q_{2}$ and $q_{3}$, or the threshold coalescence parameter $f_{c}$, introduced in the GTN model), and the identification of these parameters is not always easy and often questionable. Moreover, and despite the significant progress made in this area, these extended models are still unable to accurately consider relatively complicated situations, such as complex loadings (as these models are mainly based on axisymmetric loading), or realistic void shapes (as these models only consider spherical or ellipsoidal voids).

To overcome the above-mentioned drawbacks, a number of numerical approaches, based on unit cell finite element computations, have been developed in the literature. In these models, the ductile solid is represented by a spatially periodic arrangement of identical unit cells. Therefore, to describe the mechanical behavior of 
the whole solid, it is sufficient to consider a single unit cell, to which are applied relevant boundary conditions that accurately account for the effect of neighboring unit cells on the mechanical behavior (generally periodic or kinematic boundary conditions or a combination of them). Thanks to its reliability and flexibility, unit cell analysis has been widely employed to investigate the mechanical response of voided materials as well as the competition between the phenomena of void coalescence and strain localization. To thoroughly analyze this competition, it is essential to couple unit cell computations with relevant theoretical criteria and indicators that are able to accurately predict such material instability phenomena. Several indicators have been adopted in some contributions as void coalescence criteria, while in other contributions as strain localization criteria. Indeed, the distinction between the two phenomena and the corresponding criteria has not been clearly established in early investigations. These criteria can be categorized into four main families:

- Initial imperfection criteria: this approach, following the same spirit as the Marciniak and Kuczynski method (Marciniak and Kuczynski, 1967), assumes that strain localization occurs when the ratio $\eta$ of the deformation gradient rate inside the unit cell to that outside the unit cell becomes sufficiently large. It has been first introduced by Needleman and Tvergaard (1992) within unit cell computations to predict the onset of strain localization. This indicator has subsequently been adopted by Dunand and Mohr (2014), Daehli et al. (2017) and Zhu et al. (2018) to predict the onset of void coalescence. In Dunand and Mohr (2014) and Daehli et al. (2017), the critical value of parameter $\eta$ has been set to 5.0. However, Zhu et al. (2018) have set the critical value of $\eta$ to 10.0 by following the work of Barsoum and Faleskog (2011). The above investigations reveal the difficulty in defining a unified and consistent threshold value for $\eta$. Moreover, the associated numerical predictions are generally sensitive to the mesh refinement, and this approach is not able to predict void coalescence for high stress triaxiality, and when the Lode parameter is close to 0, as demonstrated in Barsoum and Faleskog (2011). A very similar criterion has been used in Tekoğlu et al. (2015) to predict the onset of strain localization in voided ductile solids.

- Maximum load criteria: this class of criteria has been initiated by Tvergaard (2012) and recently used by Tekoğlu et al. (2015), who have assumed that strain localization occurs when the equivalent macroscopic stress reaches its maximum value. More recently, Guo and Wong (2018) have proposed a strain localization indicator, which assumes that strain localization is met when the macroscopic force applied on the unit cell reaches its maximum value. The same authors have demonstrated that this criterion is equivalent to the Rice bifurcation approach (Rudnicki and Rice, 1975; Rice, 1976). It is interesting to note that this approach is somehow similar to the maximum force criterion developed by Swift (1952) to predict the occurrence of diffuse necking in thin metal sheets.

- Energy-based criterion: this approach, which has been initiated by Wong and Guo (2015), is exclusively adopted to predict the onset of void coalescence. It defines void coalescence as the point along the 
straining history where the ratio of overall elastic to plastic work rates of the unit cell attains a negative minimum value. This energy-based criterion has recently been utilized in several investigations to predict the onset of void coalescence (Liu et al., 2016; Dæhli et al., 2017; Luo and Gao, 2018; Guo and Wong, 2018).

- Void growth type criteria: this family of criteria assumes that void coalescence occurs when void growth exhibits abrupt acceleration. Tekoğlu et al. (2015) have developed an indicator by closely following the same concept, which assumes that the onset of void coalescence is reached when the ratio of the maximum to the minimum effective plastic strain rate at the void surface first exceeds 15.0.

It is well known that the competition between the phenomena of macroscopic strain localization and void coalescence is generally dependent on the stress state, especially the stress triaxiality ratio $T$ and the Lode parameter $\boldsymbol{L}$. Tekoğlu et al. (2015) have demonstrated that macroscopic strain localization occurs prior to void coalescence at high stress triaxiality, while at lower stress triaxiality, the two phenomena occur simultaneously. Motivated by this latter investigation, Guo and Wong (2018) have shown that the onset of macroscopic strain localization and that of void coalescence are distinct, and that macroscopic strain localization plays a precursor role to void coalescence. Furthermore, they demonstrate that the difference in the strain levels corresponding to the onset of strain localization and void coalescence, respectively, decreases as stress triaxiality $T$ increases, suggesting that both phenomena may occur simultaneously for sufficiently large $T$. These latter results are at variance with the trends obtained by Tekoğlu et al. (2015). This apparent contradiction is likely to be attributable to the difference between the void coalescence and strain localization criteria used in both investigations. For the considered ranges of stress triaxiality $(0.7 \leq T \leq 2.0)$ and Lode parameter $(-1.0 \leq L \leq 1.0)$, Guo and Wong (2018) have enumerated three possible scenarios associated with different ranges of $T$ and $L$ : both macroscopic strain localization and void coalescence are possible (for $1.0 \leq T \leq 2.0$, independently of the value of $L$ ); macroscopic strain localization is possible, but void coalescence is not possible (for $0.8 \leq T \leq 0.9$ and $-1.0 \leq L \leq 0.4$ ); both macroscopic strain localization and void coalescence are not possible (for $T=0.7$ and $-1.0 \leq L \leq 1.0$ ).

In the present paper, unit cell computations are performed to investigate the competition between macroscopic strain localization and void coalescence for a wide range of loading states. The unit cell is subjected to fully periodic boundary conditions ( $\mathrm{PBCs}$ ), allowing for the accurate modeling of the interaction between the studied unit cell and the neighboring ones. This point represents the first main theoretical originality of the developed approach, as compared to the earlier ones. The periodic homogenization multiscale scheme is used for determining the macroscopic behavior of the unit cell. This multiscale scheme is coupled with the condensation technique, developed in Miehe (2003), to numerically evaluate the macroscopic tangent modulus relating the macroscopic nominal stress rate to the macroscopic velocity gradient. The determination of the macroscopic 
tangent modulus allows rigorously applying the loss of ellipticity criterion (Rudnicki and Rice, 1975; Rice, 1976), and hence the prediction of the onset of macroscopic strain localization via the bifurcation theory. This accurate application of the loss of ellipticity criterion constitutes the second main theoretical originality of our approach, as the earlier numerical approaches were not able to determine the macroscopic tangent modulus. The competition between the onset of strain localization predicted by the Rice bifurcation theory and the ductility limits predicted by other existing criteria is investigated. To analyze this competition, attention is focused on two main configurations of loading states. Firstly, loadings under proportional stressing (or constant stress paths) are considered, where the stress triaxiality ratio $T$ ranges between 0.7 and 3.0, and the Lode parameter $L$ is comprised between -1.0 and 1.0. For this first loading configuration, our numerical predictions are found to be consistent with the classical published trends: strain localization occurs prior to void coalescence, both being predicted at realistic strain levels for the whole ranges of $T$ and $L$. Moreover, the trends obtained in Guo and Wong (2018), stating that the difference between the strain levels corresponding to the onset of strain localization and void coalescence decreases as stress triaxiality $T$ increases, are confirmed by our numerical predictions. The second loading configuration covers the in-plane strain paths used for predicting the forming limit diagrams (i.e., from uniaxial to equibiaxial tension states). Although of major importance in the context of forming processes (formability of thin metal sheets), this second loading configuration has not been sufficiently investigated in the early studies based on unit cell computations. Our numerical predictions reveal that only plastic strain localization may occur for this second configuration of loading, as void coalescence cannot be reached. The developed approach, based on the coupling between the periodic homogenization scheme and the strain localization and coalescence criteria, is also used for investigating the effects of void shape and secondary population of voids on the ductility limit of thin metal sheets.

This paper is organized as follows:

- Section 2 details the micromechanical approach used to model the unit cell behavior.

- Section 3 presents the boundary conditions and the two macroscopic loading configurations applied in the unit cell computations.

- The adopted strain localization and void coalescence criteria are described in Section 4.

- The numerical results of the current study are reported and extensively discussed in Section 5.

- Section 6 closes our contribution by summarizing some conclusions and future works.

\section{Conventions, notations and abbreviations}

The following conventions and notations are used throughout the paper:

- Microscale (resp. macroscale) variables are denoted by lowercase (resp. capital) letters. 
- Vectors and tensors are indicated by bold letters or symbols. By contrast, scalar parameters and variables are designated by thin and italic letters or symbols.

- Einstein's convention of implied summation over repeated indices is adopted. The range of free (resp. dummy) index is given before (resp. after) the corresponding equation.

- $\quad$ time derivative of $\bullet$.

- $\quad$ transpose of $\bullet$.

- $\bullet^{-1} \quad$ inverse of $\bullet$.

- $\operatorname{sgn}(\bullet) \quad \operatorname{sign}$ of $\bullet$.

- $\operatorname{tr}(\bullet) \quad$ trace of $\bullet$.

- $\operatorname{det}(\bullet) \quad$ determinant of $\bullet$.

- $\ln (\bullet) \quad$ natural logarithm of $\bullet$.

- $|\bullet| \quad$ absolute value of $\bullet$.

- $\mathbf{I}_{2}$ second-rank identity tensor.

- DOF degree of freedom.

- MPC multi-point constraints option (ABAQUS terminology).

- PBCs periodic boundary conditions.

- KBCs kinematic boundary conditions.

\section{Micromechanical modeling of the unit cell}

We consider a ductile solid defined as an array of cubic unit cells containing a void at their center, as shown in Fig. 1a. Each unit cell may be regarded as a heterogeneous medium composed of two main phases: the primary void and the metal matrix, which is itself assumed to be voided to account for the possible effect of secondary population of voids (Fig. 1b). The initial shape of the primary void is assumed to be spherical or ellipsoidal, while all the secondary voids are assumed to be spherical. A Cartesian frame $\left(\overrightarrow{\mathbf{e}}_{1}, \overrightarrow{\mathbf{e}}_{2}, \overrightarrow{\mathbf{e}}_{3}\right)$ is introduced to define the coordinates of the material points, where vectors $\overrightarrow{\mathbf{e}}_{i}$ are normal to the faces of the unit cell in the initial configuration. The origin of this coordinate system is located at the center of the unit cell. Hence, the initial unit cell occupies the domain $\left[-l_{0} / 2, l_{0} / 2\right] \times\left[-l_{0} / 2, l_{0} / 2\right] \times\left[-l_{0} / 2, l_{0} / 2\right]$, as shown in Fig. $1 \mathrm{~b}$ (with $\left.l_{0}=1 \mathrm{~mm}\right)$. 


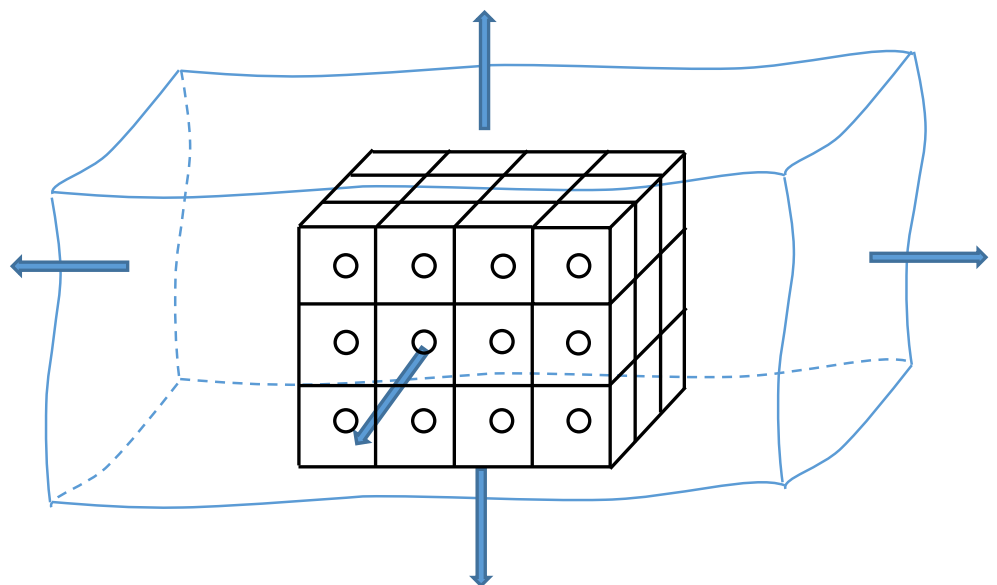

(a)

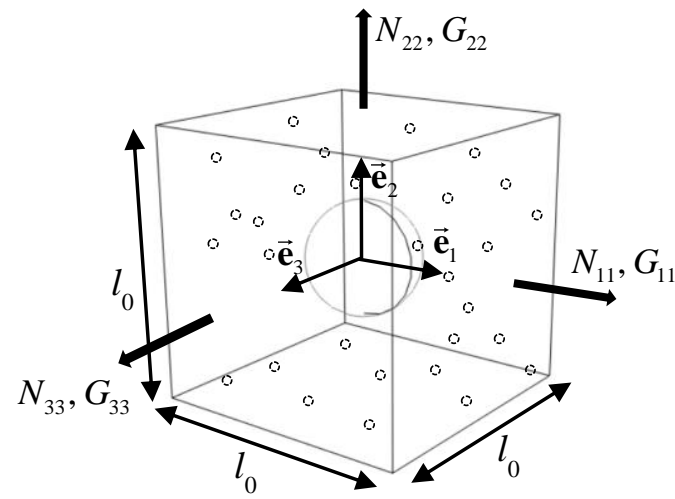

(b)

Fig. 1. (a) Micromechanical model of a material layer composed of an arrangement of cubic voided unit cells; (b) A unit cell containing a centered, spherical void surrounded by a voided matrix.

Considering the periodicity of the void arrangement (Fig. 1a), the periodic homogenization seems to be a suitable multiscale scheme to determine the homogenized behavior of the unit cell (Miehe, 2003; Zhu et al., 2020). The use of this homogenization technique allows substituting the heterogeneous unit cell by an equivalent homogenized medium with the same effective mechanical properties (Fig. 2).

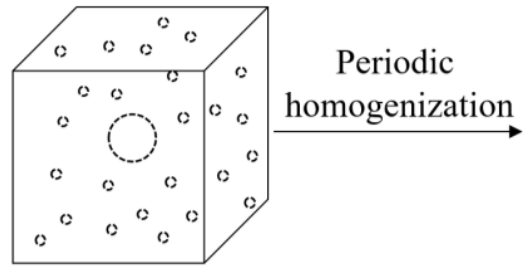

(a)

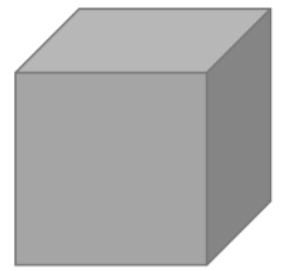

(b)

Fig. 2. Illustration of the concept of periodic homogenization: (a) unit cell containing primary and secondary voids; (b) equivalent homogenized medium.

The periodic homogenization equations, formulated using Eulerian variables, are summarized as follows:

- The microscopic velocity gradient $\mathrm{g}$, which is additively decomposed into its macroscopic counterpart $\mathbf{G}$ and a fluctuation velocity gradient $\mathbf{g}^{\text {per }}$ :

$$
\mathbf{g}=\mathbf{G}+\mathbf{g}^{\text {per }}
$$

where $\mathbf{g}^{\text {per }}$ is periodic over the boundary of the unit cell (in its current configuration).

By spatial integration of Eq. (1), the velocity $\dot{\mathbf{x}}$ of a microscopic material point can be expressed as a function of its current position $\mathbf{x}$ and a periodic velocity field $\mathbf{v}^{\text {per }}$ : 


$$
\dot{\mathbf{x}}=\mathbf{G} \cdot \mathbf{X}+\mathbf{v}^{\text {per }}
$$

Further details on the practical aspects related to the application of the PBCs on the outer surfaces of the unit cell can be found in Appendix B.

It is interesting to note that kinematic boundary conditions (KBCs) can be considered as a particular case of the PBCs, where the periodic field $\mathbf{v}^{\text {per }}$ is set to zero over the outer surface of the unit cell.

- The averaging relationships that allow relating the macroscopic velocity gradient $\mathbf{G}$ and the macroscopic nominal stress rate $\dot{\mathbf{N}}$ to their microscopic counterparts $\mathbf{g}(\mathbf{x})$ and $\dot{\mathbf{n}}(\mathbf{x})$ :

$$
\mathbf{G}=\frac{1}{|V|} \int_{V} \mathbf{g}(\mathbf{x}) d V \quad ; \quad \dot{\mathbf{N}}=\frac{1}{|V|} \int_{V} \dot{\mathbf{n}}(\mathbf{x}) d V
$$

where $V$ represents the current volume of the unit cell.

Practically, the unit cell is subjected to the macroscopic velocity gradient $\mathbf{G}$, or the macroscopic nominal stress rate $\dot{\mathbf{N}}$, or complementary components of them. The developments of Sections 3.1 and 3.2 provide more details on how to apply such a macroscopic loading.

- The constitutive relation at the macroscopic scale, relating the macroscopic nominal stress rate $\dot{\mathbf{N}}$ to the macroscopic velocity gradient $\mathbf{G}$ via the macroscopic tangent modulus $\mathcal{L}$ :

$$
\dot{\mathbf{N}}=\mathcal{L}: \mathbf{G} \text {. }
$$

- The microscopic static equilibrium equation in the absence of body forces:

$$
\operatorname{div}_{\mathbf{x}}\left(\dot{\mathbf{n}}^{T}(\mathbf{x})\right)=\mathbf{0}
$$

- The constitutive relations describing the mechanical behavior of the metal matrix, which will be detailed in Appendix A.

\section{Periodic boundary conditions and macroscopic loading}

The periodic homogenization problem presented in Section 2 is solved within the ABAQUS/Standard finite element software. The main steps of this solution strategy are summarized hereafter:

- Discretization of the unit cell by finite elements: to this end, the C3D20 element (20-node quadratic brick element with full integration) is used in all the simulations except those reported in Fig. 4, which are obtained using the C3D8 element (8-node linear brick element with full integration). A higher mesh density is adopted around the primary void to avoid potential element distortion. A user-defined material (UMAT) subroutine is used for implementing the Gurson constitutive equations describing the mechanical behavior of the metal matrix. 
- Application of the periodic boundary conditions: this task is automatically managed by using the set of python scripts Homtools developed by Lejeunes and Bourgeois (2011). These periodic boundary conditions are applied on the six (resp., four) outer faces of the unit cell, when this unit cell is subjected to proportional stressing (resp., proportional in-plane strain path), as will be detailed in Sections 3.1 and 3.2. Further practical details on the application of the periodic boundary conditions will be provided in Appendix B.

- Application of macroscopic loading: in the current investigation, the unit cell may be subjected to two different loading configurations. Firstly, macroscopic proportional stressing (i.e., proportional stress paths) to investigate the effect of the stress triaxiality ratio $T$ and Lode parameter $L$ on the competition between void coalescence and macroscopic plastic strain localization. Secondly, macroscopic proportional in-plane strain paths to predict forming limit diagrams (FLDs) of thin voided sheets. To apply the first loading configuration, some extensions of the set of python scripts Homtools are required. However, the application of the second loading configuration is easily achieved by using the Homtools. Further details on the first and second loading configuration will be given in Sections 3.1 and 3.2, respectively.

- Computation of the macroscopic mechanical response: the Homtools enables to readily and automatically manage this task.

\subsection{Proportional stressing}

As previously stated, loadings under macroscopic proportional stressing (i.e., proportional stress paths) are applied to investigate the effect of the stress triaxiality ratio $T$ and Lode parameter $L$ on the competition between void coalescence and macroscopic plastic strain localization. In this case, the unit cell presented in Fig. $1 \mathrm{~b}$ is subjected to a diagonal triaxial macroscopic stress state (without shear stresses), where only components $N_{11}, N_{22}$, and $N_{33}$ of the nominal stress tensor $\mathbf{N}$ are different from zero.

Proportional stress state requires that the stress ratios $\beta_{1}$ and $\beta_{2}$ defined as:

$$
\beta_{1}=\frac{\Sigma_{11}}{\Sigma_{33}} ; \beta_{2}=\frac{\Sigma_{22}}{\Sigma_{33}},
$$

should be kept constant during the deformation history. In Eq. (6), $\Sigma_{11}, \Sigma_{22}$ and $\Sigma_{33}$ designate the diagonal components of the macroscopic Cauchy stress tensor $\boldsymbol{\Sigma}$, which is related to its microscopic counterpart $\boldsymbol{\sigma}$ through the following averaging rule:

$$
\mathbf{\Sigma}=\frac{1}{|V|} \int_{V} \boldsymbol{\sigma}(\mathbf{x}) d V
$$


The macroscopic hydrostatic stress $\Sigma_{h}$ and the macroscopic equivalent (von Mises) stress $\Sigma_{e q}$ are obtained from components $\Sigma_{11}, \Sigma_{22}$ and $\Sigma_{33}$ as:

$$
\Sigma_{h}=\frac{\Sigma_{11}+\Sigma_{22}+\Sigma_{33}}{3} ; \quad \Sigma_{e q}=\frac{1}{\sqrt{2}} \sqrt{\left(\Sigma_{11}-\Sigma_{22}\right)^{2}+\left(\Sigma_{11}-\Sigma_{33}\right)^{2}+\left(\Sigma_{22}-\Sigma_{33}\right)^{2}} .
$$

Assuming that $\Sigma_{11} \geq \Sigma_{22} \geq \Sigma_{33}$, the macroscopic stress triaxiality ratio $T$ and Lode parameter $L$ can be expressed in terms of the stress ratios $\beta_{1}$ and $\beta_{2}$ (Liu et al., 2016):

$$
\left\{\begin{array}{l}
T=\frac{\Sigma_{h}}{\Sigma_{e q}}=\frac{\sqrt{2}\left(1+\beta_{1}+\beta_{2}\right)}{3 \sqrt{\left(1-\beta_{1}\right)^{2}+\left(1-\beta_{2}\right)^{2}+\left(\beta_{1}-\beta_{2}\right)^{2}}} \operatorname{sgn}\left(\Sigma_{33}\right) ; \\
L=\frac{\left(2 \beta_{2}-\beta_{1}-1\right)}{\beta_{1}-1}, \quad-1 \leq L \leq 1 .
\end{array}\right.
$$

Stress triaxiality ratio $T$ and Lode parameter $L$ characterize the spherical and deviatoric parts of the macroscopic stress state, respectively. Ratios $T$ and $L$ are kept constant during the deformation history by prescribing constant values for $\beta_{1}$ and $\beta_{2}$. By inverting Eq. (9), $\beta_{1}$ and $\beta_{2}$ can be expressed as functions of $T$ and $L$ :

$$
\beta_{1}=\frac{3 T \sqrt{3+L^{2}}+3-L}{3 T \sqrt{3+L^{2}}-3-L} \quad ; \quad \beta_{2}=\frac{3 T \sqrt{3+L^{2}}+2 L}{3 T \sqrt{3+L^{2}}-3-L} .
$$

It is worthwhile to note that this inversion is not unique, as multiple combinations of $\beta_{1}$ and $\beta_{2}$ can be obtained for the same values of $T$ and $L$ (Wong and Guo, 2015). By following Liu et al. (2016), the solution for $\beta_{1}$ and $\beta_{2}$ given by Eq. (10) is adopted for all the predictions of Section 5.3. Meanwhile, we have adopted the following sign convention for $L$ : the extreme values of $L=+1,-1,0$ refer to the stress state case of generalized compression, generalized tension and pure shear, superimposed with hydrostatic stress, respectively (Liu et al., 2016). Note that an opposite sign convention, $L=+1,-1$, is adopted in numerous studies (Dunand and Mohr, 2014; Guo and Wong, 2018; Wong and Guo, 2015) for generalized tension and generalized compression, resectively).

To apply proportional triaxial stressing, 3D periodic boundary conditions shall be imposed on the six outer faces of the unit cell (two by two faces), following the concept presented in Appendix B. In this case, three reference points $R P_{1}, R P_{2}$ and $R P_{3}$ are created by using the Homtools to manage these boundary conditions and the macroscopic loading. These reference points are defined by the following displacements: 


$$
\begin{aligned}
& R P_{1}: U_{11}=\left(F_{11}-1\right) l_{0} ; U_{12}=0 ; U_{13}=0 ; \\
& R P_{2}: U_{21}=0 ; U_{22}=\left(F_{22}-1\right) l_{0} ; U_{23}=0 ; \\
& R P_{3}: U_{31}=0 ; U_{32}=0 ; U_{33}=\left(F_{33}-1\right) l_{0} .
\end{aligned}
$$

Components $F_{11}, F_{22}$ and $F_{33}$ of the macroscopic deformation gradient $\mathbf{F}$ should be prescribed in such a way that the stress triaxiality ratio $T$ and the Lode parameter $L$ hold constant during the entire deformation history. To conveniently apply the prescribed macroscopic deformation gradient $\mathbf{F}$, an extra dummy (or 'ghost') node is introduced into the finite element model. The DOFs of this dummy node and the associated reaction forces are denoted $\left(U_{1}^{*}, U_{2}^{*}, U_{3}^{*}\right)$ and $\left(\alpha_{1}, \alpha_{2}, \alpha_{3}\right)$, respectively. A user subroutine MPC (ABAQUS, 2014) has been developed to connect the dummy node to the three reference points $R P_{1}, R P_{2}$ and $R P_{3}$ (and further to the unit cell). In this subroutine, the reference points serve as slave nodes, while the dummy node serves as master node wherein the loading is imposed. The master node transmits the imposed loading through the multi-point constraints to the reference points as stated by Eq. (12):

$$
\left[\begin{array}{l}
U_{11} \\
U_{22} \\
U_{33}
\end{array}\right]=\mathcal{T}\left[\begin{array}{c}
U_{1}^{*} \\
U_{2}^{*} \\
U_{3}^{*}
\end{array}\right]
$$

where $\mathcal{T}$ is a functional to be determined in order to ensure that the displacements $U_{11}, U_{22}$, and $U_{33}$ applied on the reference points lead to the prescribed ratios $\beta_{1}$ and $\beta_{2}$ between the different macroscopic stress components. A simplified illustration of the MPC subroutine is shown in Fig. 3.

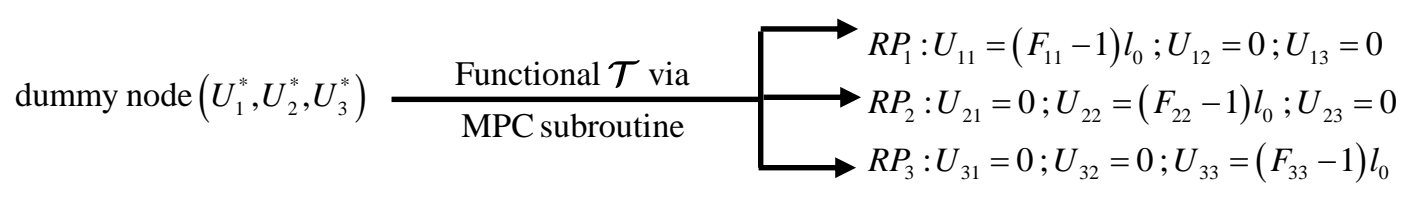

Fig. 3. Schematic illustration of the multi-point constraints between the dummy node and the reference points

$$
\left(R P_{1}, R P_{2}, R P_{3}\right) \text {. }
$$

We next detail the derivation of the expression of functional $\mathcal{T}$. In this aim, the work rate equivalence between the dummy node and the unit cell shall be used (Liu et al., 2016):

$$
\boldsymbol{\alpha}^{T} \cdot \dot{\mathbf{U}}^{*}=|V| \tilde{\boldsymbol{\Sigma}}^{T} \cdot \tilde{\mathbf{G}}
$$

where $\mathbf{U}^{*}$ and $\boldsymbol{\alpha}$ are the displacement and the associated reaction force vectors of the dummy node, respectively. As to $\tilde{\boldsymbol{\Sigma}}$ and $\tilde{\mathbf{G}}$, they represent the storage vectors for the diagonal components of the macroscopic Cauchy stress and the macroscopic velocity gradient associated with the unit cell, respectively: 


$$
\boldsymbol{\alpha}=\left[\begin{array}{l}
\alpha_{1} \\
\alpha_{2} \\
\alpha_{3}
\end{array}\right] ; \quad \mathbf{U}^{*}=\left[\begin{array}{l}
U_{1}^{*} \\
U_{2}^{*} \\
U_{3}^{*}
\end{array}\right] ; \quad \tilde{\boldsymbol{\Sigma}}=\left[\begin{array}{c}
\Sigma_{11} \\
\Sigma_{22} \\
\Sigma_{33}
\end{array}\right] ; \quad \tilde{\mathbf{G}}=\left[\begin{array}{c}
G_{11} \\
G_{22} \\
G_{33}
\end{array}\right]
$$

Vectors $\dot{\mathbf{U}}^{*}$ and $\tilde{\mathbf{G}}$ may be linked by a transformation matrix $\mathcal{R}$ belonging to $\mathcal{S O}(3)^{1}$ (Wong and Guo, 2015):

$$
\left[\begin{array}{c}
G_{11} \\
G_{22} \\
G_{33}
\end{array}\right]=\mathcal{R} \cdot\left[\begin{array}{c}
\dot{U}_{1}^{*} \\
\dot{U}_{2}^{*} \\
\dot{U}_{3}^{*}
\end{array}\right]
$$

The external loading is applied on the dummy node and the transformation matrix is used to suitably transfer this loading on the different reference points. We apply a linear displacement on only the third DOF of the dummy node with $\dot{U}_{3}^{*}=1$. The first two DOFs are left free. Consequently, the corresponding reaction forces are equal to zero (namely, $\alpha_{1}=\alpha_{2}=0$ ). With this particular loading, Eq. (13) reduces to:

$$
\alpha_{3} \dot{U}_{3}^{*}=|V|\left(\Sigma_{11} G_{11}+\Sigma_{22} G_{22}+\Sigma_{33} G_{33}\right) .
$$

Without dwelling into the mathematical details, which have been extensively discussed in Wong and Guo (2015) and Liu et al. (2016), the expression of $\alpha_{3}$ can be derived, by involving Eqs. (6), as follows:

$$
\alpha_{3}=|V| \sqrt{\left(\Sigma_{11}\right)^{2}+\left(\Sigma_{22}\right)^{2}+\left(\Sigma_{33}\right)^{2}}=|V|\left(\sqrt{\left(\beta_{1}\right)^{2}+\left(\beta_{2}\right)^{2}+1}\right) \Sigma_{33} .
$$

Using the fact that $\alpha_{1}=\alpha_{2}=0$, the transformation matrix $\mathcal{R}$ can be expressed by following the formulation given in Liu et al. (2016):

$$
\mathcal{R}=\left[\begin{array}{ccc}
\frac{\left(\beta_{1}\right)^{2} \mu+\left(\beta_{2}\right)^{2}}{\left(\beta_{1}\right)^{2}+\left(\beta_{2}\right)^{2}} & \frac{\beta_{1} \beta_{2}(\mu-1)}{\left(\beta_{1}\right)^{2}+\left(\beta_{2}\right)^{2}} & \beta_{1} \mu \\
\frac{\beta_{1} \beta_{2}(\mu-1)}{\left(\beta_{1}\right)^{2}+\left(\beta_{2}\right)^{2}} & \frac{\left(\beta_{1}\right)^{2}+\left(\beta_{2}\right)^{2} \mu}{\left(\beta_{1}\right)^{2}+\left(\beta_{2}\right)^{2}} & \beta_{2} \mu \\
-\beta_{1} \mu & -\beta_{2} \mu & \mu
\end{array}\right] \text { with } \mu=\frac{1}{\sqrt{\left(\beta_{1}\right)^{2}+\left(\beta_{2}\right)^{2}+1}} .
$$

The form given by Eq. (18) for the transformation matrix $\mathcal{R}$ is valid only for $\Sigma_{33}>0$ (i.e., $\operatorname{sgn}\left(\Sigma_{33}\right)=1$ ). This condition is obviously ensured for the loadings studied in Section 5.3, where $0.7 \leq T \leq 3$ and $-1 \leq L \leq 1$, which corresponds to positive stress ratios $\beta_{1}$ and $\beta_{2}$.

\footnotetext{
${ }^{1}$ Matrix $\mathcal{R}$ belongs to $\mathcal{S O}(3)$ if $\mathcal{R}$ is orthogonal (i.e., $\mathcal{R}^{-1}=\mathcal{R}^{T}$ ) and $\operatorname{det}(\mathcal{R})=1$.
} 
For proportional stressing, the transformation matrix $\mathcal{R}$ holds constant during the loading (as ratios $\beta_{1}$ and $\beta_{2}$ do not change). Hence, the integration of Eq. (15) leads to the following expression:

$$
\left[\begin{array}{l}
E_{11} \\
E_{22} \\
E_{33}
\end{array}\right]=\mathcal{R} \cdot\left[\begin{array}{l}
U_{1}^{*} \\
U_{2}^{*} \\
U_{3}^{*}
\end{array}\right],
$$

where $\mathbf{E}$ is the macroscopic logarithmic strain tensor defined as:

$$
\mathbf{E}=\int_{0}^{t} \mathbf{G} d t=\ln (\mathbf{F})
$$

The combination of Eqs. (11), (19) and (20) yields:

$$
\left\{\begin{array}{l}
F_{11}=\left(U_{11} / l_{0}\right)+1=e^{\left(\mathcal{R}_{11} U_{1}^{*}+\mathcal{R}_{12} U_{2}^{*}+\mathcal{R}_{13} U_{3}^{*}\right)} \\
F_{22}=\left(U_{22} / l_{0}\right)+1=e^{\left(\mathcal{R}_{21} U_{1}^{*}+\mathcal{R}_{22} U_{2}^{*}+\mathcal{R}_{23} U_{3}^{*}\right)} \\
F_{33}=\left(U_{33} / l_{0}\right)+1=e^{\left(\mathcal{R}_{31} U_{1}^{*}+\mathcal{R}_{32} U_{2}^{*}+\mathcal{R}_{33} U_{3}^{*}\right)}
\end{array}\right.
$$

The expression of functional $\mathcal{T}$ can be readily identified from Eq. (21). Thus, the relations between the DOFs of the dummy node and those of the three reference points to be implemented in the MPC user subroutine are summarized by Eqs. (21). The periodic boundary conditions together with constraints (21) determine the boundary value problem of the unit cell, and the proportional stressing applied during the loading history.

\subsection{Proportional in-plane strain paths}

We consider a thin metal sheet made of 2D array of voided unit cells. This sheet can be viewed as a particular case of Fig. 1(a), where a single unit cell is considered in the thickness direction. Loading under macroscopic proportional in-plane strain paths is classically adopted to predict forming limit diagrams (FLDs) of thin metal sheets. In this case, the unit cell is subjected to biaxial stretching in the 1 and 2 directions (Fig. 1b). Additionally, the out-of-plane components of the macroscopic nominal stress $\mathbf{N}$ (and thus $\mathbf{\Sigma}$ ): $N_{13}, N_{23}, N_{31}, N_{32}$ and $N_{33}$ are set to zero. The strain-path ratio $\rho=G_{22} / G_{11}$ is kept constant during the loading, and it ranges between $-1 / 2$ (uniaxial tension state) and 1 (equibiaxial tension state). The other in-plane components of the macroscopic velocity gradient $\left(G_{12}, G_{21}\right)$ are set to zero. In this case, periodic boundary conditions are only applied on the faces normal to directions 1 and 2 (Fig. 1b). However, faces normal to direction 3 are free from any boundary condition. This specific choice enables to ensure the macroscopic plane-stress state in the third direction.

In terms of Eulerian measures, the macroscopic loading takes the following form: 


$$
\mathbf{G}=\left[\begin{array}{ccc}
G_{11} & 0 & ? \\
0 & \rho G_{11} & ? \\
? & ? & ?
\end{array}\right] \quad ; \quad \dot{\mathbf{N}}=\left[\begin{array}{ccc}
? & ? & 0 \\
? & ? & 0 \\
0 & 0 & 0
\end{array}\right]
$$

where components marked by '?' are unknown and need to be determined. Components $G_{13}, G_{31}, G_{23}, G_{32}$ and $G_{33}$ are calculated by making use of the plane-stress conditions:

$$
\dot{N}_{13}=\dot{N}_{31}=\dot{N}_{23}=\dot{N}_{32}=\dot{N}_{33}=0 .
$$

Considering the void symmetry and the isotropic behavior of the matrix, the following equivalence holds:

$$
\dot{N}_{13}=\dot{N}_{31}=\dot{N}_{23}=\dot{N}_{32}=0 \Leftrightarrow G_{13}=G_{31}=G_{23}=G_{32}=0
$$

Under this equivalence, the macroscopic loading of Eq. (22) can be expressed in a Lagrangian formulation more suitable for the application of the Homtools:

$$
\mathbf{F}=\left[\begin{array}{ccc}
F_{11} & 0 & ? \\
0 & \left(F_{11}\right)^{\rho} & ? \\
? & ? & ?
\end{array}\right] \quad ; \quad \mathbf{P}=\left[\begin{array}{ccc}
? & ? & 0 \\
? & ? & 0 \\
0 & 0 & 0
\end{array}\right] .
$$

To apply the 2D periodic boundary conditions and the macroscopic loading of Eq. (25), three reference points $R P_{1}, R P_{2}$ and $R P_{3}$ should be created. The following prescribed boundary conditions should be applied on the reference points (a displacement on $R P_{1}$ and $R P_{2}$, and a force on $R P_{3}$ ) to comply with Eq. (25):

$$
\begin{aligned}
& R P_{1}: U_{11}=\left(F_{11}-1\right) l_{0} ; U_{12}=0 ; U_{13}=0 ; \\
& R P_{2}: U_{21}=0 ; U_{22}=\left(\left(F_{11}\right)^{\rho}-1\right) l_{0} ; U_{23}=0 ; \\
& R P_{3}: R F_{31}=0 ; R F_{32}=0 ; R F_{33}=0 .
\end{aligned}
$$

\section{Void coalescence and strain localization criteria}

In the present work, attention is directed towards the prediction of ductile failure by using four indicators, which will be presented hereafter: the first three ones have been used in previous contributions (but without rigorous coupling with the periodic homogenization multiscale scheme), while the last one is applied for the first time herein. These different indicators will be classified for the loading case under proportional stressing.

\subsection{Maximum reaction force criterion}

This indicator has been adopted in Guo and Wong (2018) to predict the onset of strain localization. With this criterion, strain localization is attained when the reaction force component $\alpha_{3}$ applied on the dummy node and defined by Eq. (17) reaches its maximum value, or equivalently: 


$$
\dot{\alpha}_{3}=0
$$

The critical equivalent strain predicted at the moment when this criterion is verified will be denoted $E_{e q}^{R}$.

\subsection{Maximum equivalent stress criterion}

This indicator, initiated by Tvergaard (2012), states that material failure occurs when the macroscopic equivalent stress $\Sigma_{e q}$ reaches its maximum value. For triaxial proportional stressing, the macroscopic Cauchy stress tensor takes the general form:

$$
\boldsymbol{\Sigma}=\left[\begin{array}{ccc}
\Sigma_{11} & 0 & 0 \\
0 & \Sigma_{22} & 0 \\
0 & 0 & \Sigma_{33}
\end{array}\right]=\Sigma_{33}\left[\begin{array}{ccc}
\beta_{1} & 0 & 0 \\
0 & \beta_{2} & 0 \\
0 & 0 & 1
\end{array}\right]
$$

In this case, the macroscopic equivalent stress $\Sigma_{e q}$ can be expressed in terms of stress ratios $\beta_{1}$ and $\beta_{2}$ as follows :

$$
\Sigma_{e q}=\sqrt{\left(\beta_{1}\right)^{2}+\left(\beta_{2}\right)^{2}-\beta_{1} \beta_{2}-\beta_{1}-\beta_{2}+1}\left|\Sigma_{33}\right| .
$$

The critical equivalent strain predicted at the moment when this criterion is verified will be denoted $E_{e q}^{S}$.

\subsection{Energy-based criterion}

The energy-based criterion has been proposed by Wong and Guo (2015) and is based on the fact that void coalescence involves localization of plastic deformation between neighboring voids, with the material outside the localization band undergoing elastic unloading (Pardoen and Hutchinson, 2000). To apply this criterion, elastic and plastic work rates should be computed:

$$
\dot{W}^{e}=\int_{V} \boldsymbol{\sigma}: \mathbf{d}^{e} d V \quad ; \quad \dot{W}^{p}=\int_{V} \boldsymbol{\sigma}: \mathbf{d}^{p} d V
$$

where $\boldsymbol{\sigma}$ is the microscopic Cauchy stress tensor, $\mathbf{d}^{e}$ and $\mathbf{d}^{p}$ are respectively the elastic and plastic parts of the deformation rate tensor. The sign of the ratio $\dot{W}^{e} / \dot{W}^{p}$ implies three different loading states: $\dot{W}^{e} / \dot{W}^{p}>0$ for a state of elastoplastic loading, $\dot{W}^{e} / \dot{W}^{p}<0$ for a state of elastic unloading, $\dot{W}^{e} / \dot{W}^{p}=0$ for a state of neutral loading. Following Wong and Guo (2015), the onset of void coalescence is deemed to occur when the ratio $\dot{W}^{e} / \dot{W}^{p}$ attains a minimum and is negative.

The critical equivalent strain predicted at the moment when this criterion is verified will be denoted $E_{e q}^{C}$. 


\subsection{Rice bifurcation criterion}

Following the Rice approach (Rudnicki and Rice, 1975; Rice, 1976), the onset of strain localization may be mathematically related to the loss of ellipticity of the macroscopic governing equations. This criterion corresponds to the singularity of the macroscopic acoustic tensor $\overrightarrow{\mathcal{N}} \cdot \mathcal{L} \cdot \overrightarrow{\mathcal{N}}$ :

$$
\operatorname{det}(\overrightarrow{\mathcal{N}} \cdot \mathcal{L} \cdot \overrightarrow{\mathcal{N}})=0
$$

where $\overrightarrow{\mathcal{N}}$ is the unit vector normal to the localization band, and $\mathcal{L}$ the macroscopic tangent modulus introduced in Eq. (4). In contrast to all the previous contributions, we have implemented in the present investigation a numerical approach to exactly compute $\mathcal{L}$, thereby allowing the rigorous application of the Rice bifurcation criterion. This approach is conceptually based on the condensation technique proposed by Miehe (2003). This technique enables to obtain the macroscopic tangent modulus $\mathcal{L}$ by condensation of the finite element global stiffness matrix $\mathbf{K}$. To implement this condensation technique, we have developed a set of python scripts that we have used for manipulating the outputs of the finite element computation. Hereafter, the main steps of this implementation, which are briefly recapitulated, while extensive practical details about this implementation can be found in Zhu et al. (2020):

- Step 1: at the end of each converged finite element increment, the global stiffness matrix $\mathbf{K}$ is assembled from the elementary ones $\mathbf{K}^{e l}$ :

$$
\mathbf{K}=\bigcup_{e l=1}^{e l=N e l} \mathbf{K}^{e l}
$$

where $\mathrm{Nel}$ designates the total number of finite elements.

- Step 2: all the nodes of the mesh are partitioned into two categories: set $\mathcal{B}$ made of the nodes located on the boundary surfaces where periodic constraints are imposed $\left(B_{1}^{-} \cup B_{1}^{+} \cup B_{2}^{-} \cup B_{2}^{+} \cup B_{3}^{-} \cup B_{3}^{+}\right.$ when proportional stressing is applied, and $B_{1}^{-} \cup B_{1}^{+} \cup B_{2}^{-} \cup B_{2}^{+}$when proportional in-plane strain path is applied), and set $\mathcal{A}$ composed of the other nodes. Using this partitioning rule, the lines and columns of the global stiffness matrix $\mathbf{K}$ are rearranged to obtain the following decomposition:

$$
\mathbf{K}=\left[\begin{array}{ll}
\mathbf{K}_{\mathcal{A} \mathcal{A}} & \mathbf{K}_{\mathcal{A B}} \\
\mathbf{K}_{\mathcal{B A}} & \mathbf{K}_{\mathcal{B B}}
\end{array}\right] .
$$

- Step 3: compute projection matrices $\mathbf{S}$ and $\mathbf{Q}$ by using the procedure provided in Zhu et al. (2020).

- Step 4: determine the macroscopic tangent modulus $\tilde{\mathcal{L}}$, relating the macroscopic first PiolaKirchhoff stress rate tensor $\dot{\mathbf{P}}$ to the deformation gradient rate tensor $\dot{\mathbf{F}}(\dot{\mathbf{P}}=\tilde{\mathcal{L}}: \dot{\mathbf{F}})$, by using the following relation: 


$$
\tilde{\mathcal{L}}=\frac{1}{\left|V_{0}\right|} \mathbf{Q} \cdot\left[\mathbf{S} \cdot\left(\mathbf{K}_{\mathcal{B B}}-\mathbf{K}_{\mathcal{B A}} \cdot \mathbf{K}_{\mathcal{A \mathcal { A }}}^{-1} \cdot \mathbf{K}_{\mathcal{A B}}\right)^{-1} \cdot \mathbf{S}^{T}\right]^{-1} \cdot \mathbf{Q}^{T}
$$

The tangent modulus $\mathcal{L}$ is obtained from $\tilde{\mathcal{L}}$ by permutation of the first two indices (Zhu et al., 2020):

$$
\forall i, j, k, l=1,2,3: \quad \mathcal{L}_{i j k l}=\tilde{\mathcal{L}}_{j k l} .
$$

The critical equivalent strain predicted at the moment when this criterion is verified will be denoted $E_{e q}^{B}$.

\section{Numerical predictions}

The material parameters used in the simulations reported in Sections 5.2, 5.3 and 5.4 are summarized in Section 5.1. Then, the validity of the periodic conditions applied on the boundary of the unit cell is examined in Section 5.2 by assessing its degree of accuracy and effectiveness in reproducing the behavior of the macroscopic medium. Afterward, the predictions performed under proportional stressing are presented in 5.3, where some of our numerical predictions are favorably compared with existing results published in the literature. The competition between void coalescence and macroscopic plastic strain localization is carefully analyzed in this section. Finally, Section 5.4 focuses on the predictions of forming limit diagrams for thin voided metal sheets by using the developed numerical approach.

\subsection{Material parameters}

The initial volume fraction of the primary void $f_{p 0}$ is set to 0.04 in all the simulations presented hereafter. The metal matrix is assumed to be fully dense for all the simulations of Sections 5.1 and 5.2. The effect of the secondary void population is investigated in Section 5.3 by varying the value of $f_{s 0}$. The mechanical behavior of the dense part of the metal matrix is assumed to be elastically and plastically isotropic. For consistent comparisons with Liu et al. (2016), the elasticity and hardening parameters provided in Table 1 are used in the different simulations.

Table 1. Elastoplastic parameters of the dense matrix.

\begin{tabular}{|c|c|c|c|c|}
\hline \multicolumn{2}{|c|}{ Elasticity } & \multicolumn{3}{c|}{ Hardening } \\
\hline$E(\mathrm{GPa})$ & $v$ & $K(\mathrm{MPa})$ & $\varepsilon_{0}$ & $n$ \\
\hline 210 & 0.3 & 958.8 & 0.0025 & 0.1058 \\
\hline
\end{tabular}

The initial yield stress $\sigma_{0}$ of the dense matrix can be deduced from the parameters given in Table 1:

$$
\sigma_{0}=K\left(\varepsilon_{0}\right)^{n} .
$$




\subsection{Validity of the periodic boundary conditions}

One of the most important issues in terms of ensuring that a homogenization multiscale scheme is accurate and effective is how the boundary conditions are treated. It is well known that KBCs (also called kinematic boundary conditions) require a large representative volume element to accurately capture microscopic properties and phenomena. By contrast, PBCs can provide better evaluations of the microscopic fields and thus of the macroscopic response than uniformly distributed conditions, even for non-periodic geometries (Kanit et al., 2003; Terada et al., 2000; Henyš et al., 2019). Despite its major importance, the effect of boundary conditions on the onset of void coalescence or macroscopic strain localization has not been analyzed in earlier investigations. In fact, a large majority of these investigations use KBCs (Liu et al., 2016) or a mixture of KBCs and PBCs (Barsoum and Faleskog, 2011; Guo and Wong, 2018; Wong and Guo, 2015; Zhu et al., 2018), and a very limited number of contributions is based on fully PBCs. We have conducted a comparative study to investigate the effect of the applied boundary conditions on the distribution of the microscopic fields in an entire sheet made of unit-cell cluster and a single unit cell constrained by PBCs or KBCs. This study confirms the reliability of PBCs compared to KBCs in the prediction of mechanical behavior of the unit cell.

Predictions of the onset of macroscopic strain localization, as given by the bifurcation theory, are then conducted on a $3 \mathrm{D}$ unit cell subjected to several proportional stressing states $(L=-1, T=0.7 ; 1.0 ; 2.0)$. In this case, the initial volume fractions $f_{p 0}$ (primary void) and $f_{s 0}$ (secondary voids) are set to 0.04 and 0.0 , respectively. As shown in Fig. 4a, a rapid drop in equivalent stress occurs as the equivalent strain reaches the critical value $E_{e q}^{B}$, indicated by full circles, when the PBCs are used (unlike the curves obtained by the KBCs). Consequently, strain localization predicted by using KBCs is expected to occur much later than that obtained by PBCs. Fig. $4 \mathrm{~b}$ shows that $E_{e q}^{B}$ obtained by KBCs is three times higher than that obtained by PBCs. This figure also demonstrates that KBCs lead to a strong overestimation of the macroscopic equivalent strain at the onset of strain localization. 


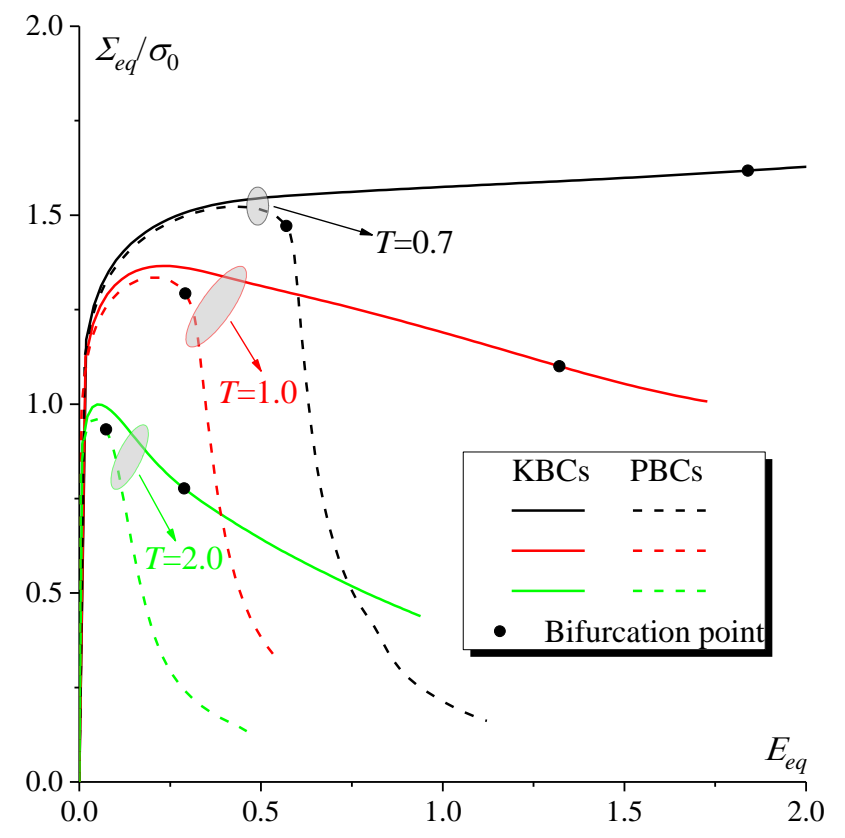

(a)

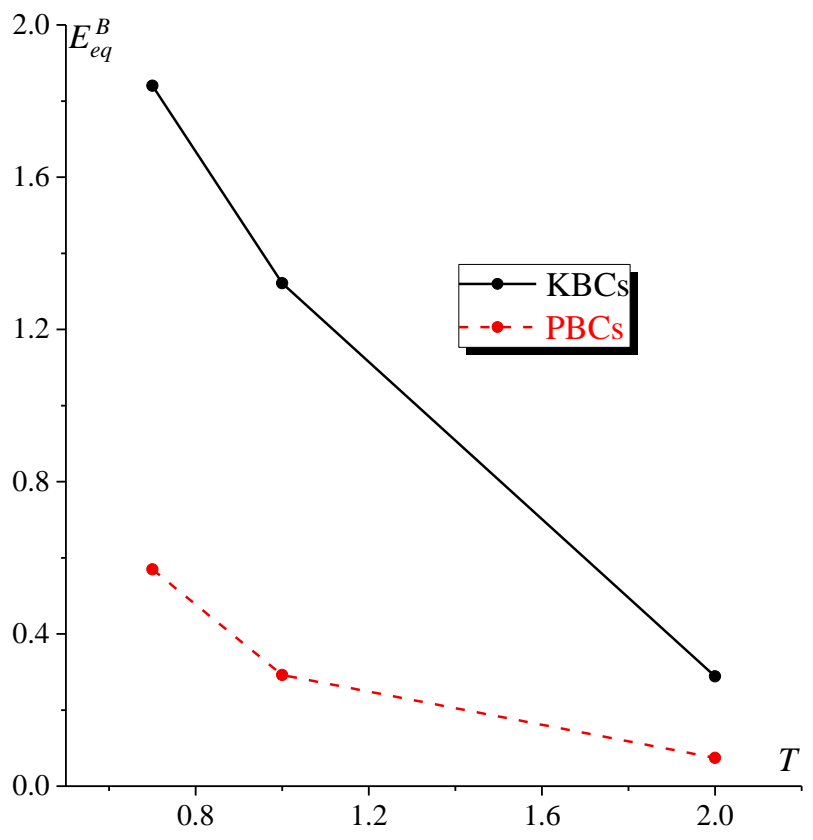

(b)

Fig. 4. Effect of the boundary conditions on: (a) the evolution of the equivalent stress $\Sigma_{\text {eq }}$ normalized by the initial yield stress $\sigma_{0} ;(\mathrm{b})$ the evolution of the critical equivalent strain $E_{e q}^{B}$.

\subsection{Proportional stressing}

\subsubsection{Comparison with results of Liu et al. (2016)}

To validate the developed approach, our numerical predictions are compared with those published in Liu et al. (2016). For this purpose, KBCs are applied, instead of PBCs, on the boundary of the unit cell. This unit cell is proportionally stressed, where the stress state is taken to be of $T=1.0$ and $L=0.0$. The onset of void coalescence is predicted by the energy-based criterion presented in Section 4.3. The evolutions of the ratio $\dot{W}^{e} / \dot{W}^{p}$, the macroscopic logarithmic strain components $\left(E_{11}, E_{22}, E_{33}\right)$ and the macroscopic equivalent stress $\Sigma_{e q}$ are plotted as functions of the macroscopic equivalent strain $E_{e q}$ in Fig. 5a, b and c, respectively. Among these plots, our numerical predictions are marked by red curves and those published in Liu et al. (2016) by grey curves. The evolution of the principal logarithmic strains and the equivalent stress-strain response are in very good agreement with those reported in Liu et al. (2016). This is also the case for the predicted value of the critical strain for void coalescence $E_{e q}^{C}\left(E_{e q}^{C}=0.597\right.$ in Liu et al., 2016 versus $E_{e q}^{C}=0.605$ in the current model). However, the magnitude of $\dot{W}^{e} / \dot{W}^{p}$ when void coalescence occurs is not in perfect agreement. This difference is likely to be attributable to the type of the finite element used in the simulations. In fact, the C3D8R element (8-node linear brick element with reduced integration) is used in Liu et al. (2016), while in the present 
investigation, the unit cell is discretized by using the C3D8 element. One can observe from the curves of Fig. 5a that, as the unit cell deforms plastically, the energy ratio undergoes a state of decrease up to a point where ratio $\dot{W}^{e} / \dot{W}^{p}$ becomes equal to zero. This means that the loading state applied on the unit cell changes from elastoplastic loading to elastic unloading. As further increase in deforming, ratio $\dot{W}^{e} / \dot{W}^{p}$ will reach a minimum value where the maximum elastic unloading occurs. This point is identified as the onset of void coalescence. Beyond this point, ratio $\dot{W}^{e} / \dot{W}^{p}$ will increase from negative to positive. This change means that the mechanical state in the unit cell recovers to elastoplastic loading from elastic unloading. Together with Fig. 5(a), Fig. 5(b) shows a shift of the principal logarithmic strains when void coalescence occurs. It is observed that the cell straining mode shifts from triaxial to uniaxial strain state with $\dot{E}_{22}=\dot{E}_{33}=0$ (Koplik and Needleman, 1988). It is worth noting that the straining mode shifts and the minimum of $\dot{W}^{e} / \dot{W}^{p}$ always occurs simultaneously and corresponds to the onset of void coalescence.

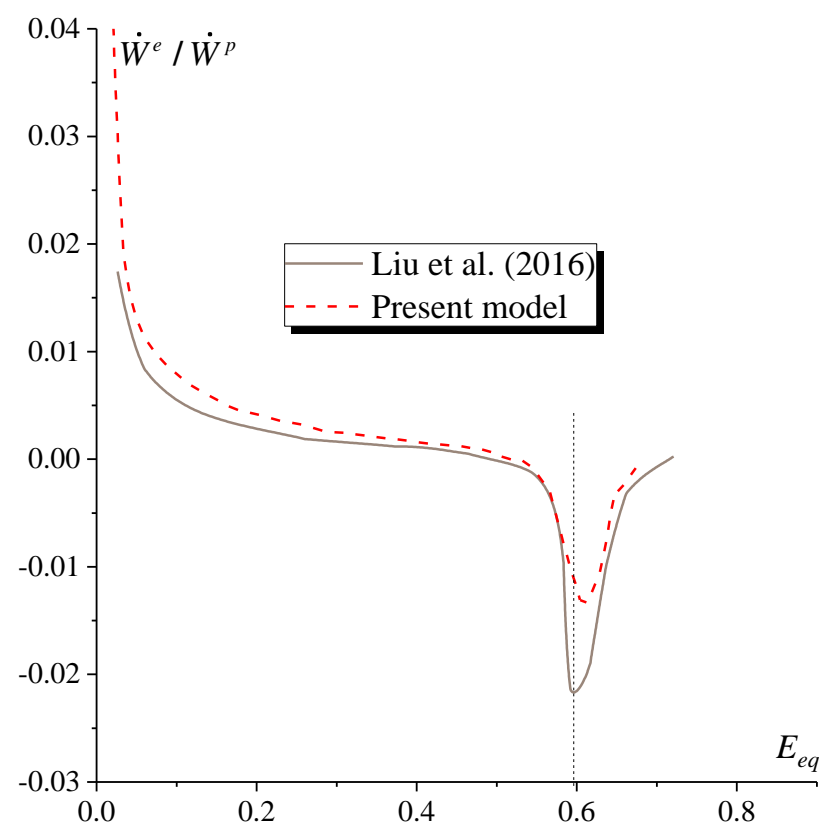

(a)

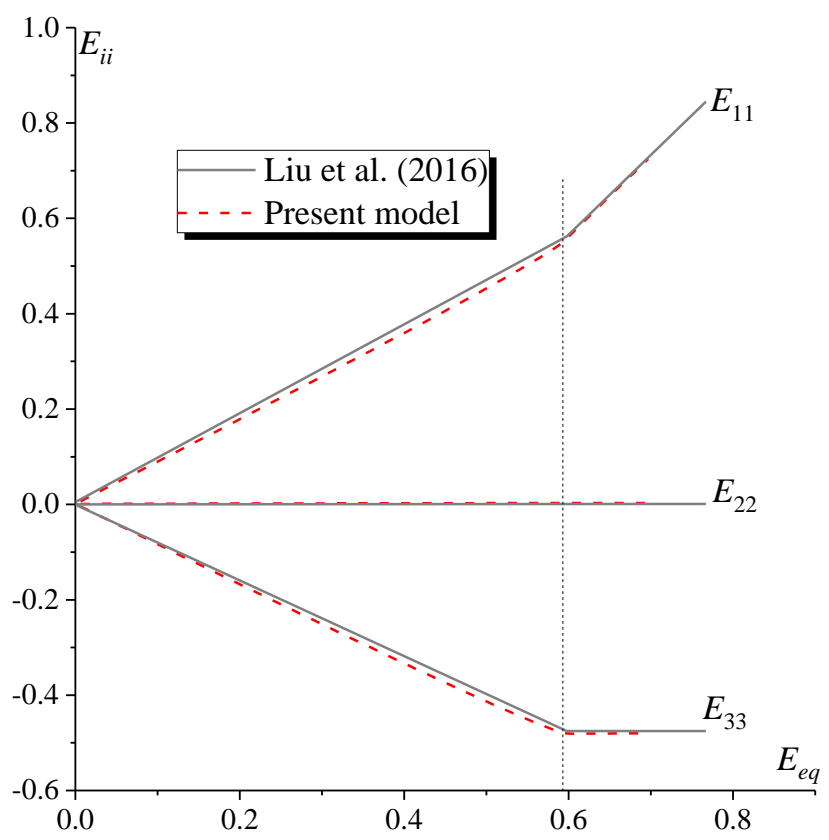

(b) 


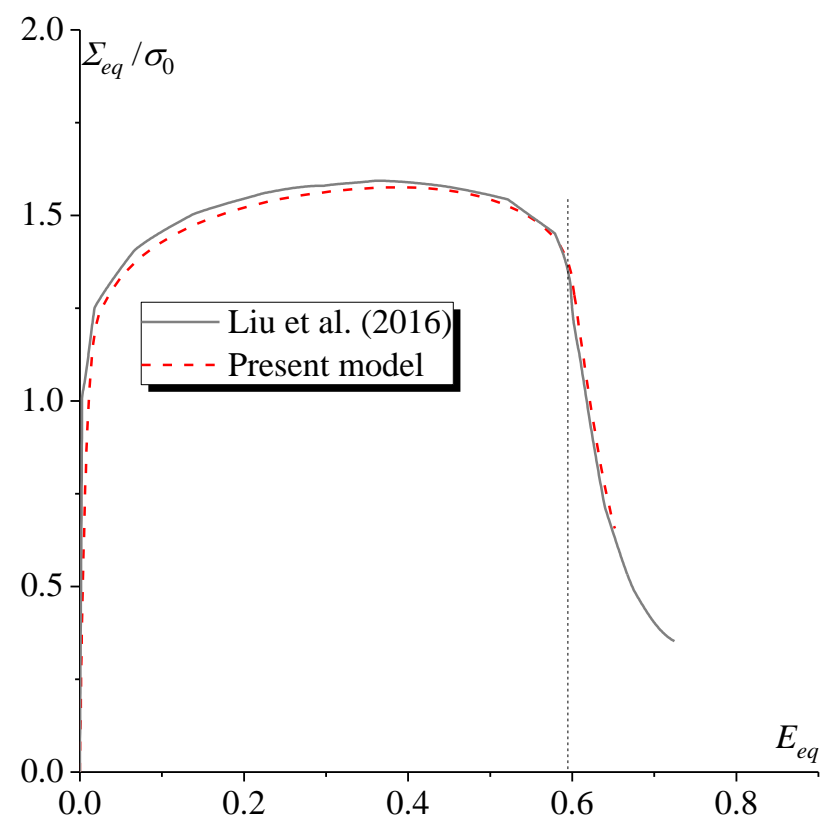

(c)

Fig. 5. Validation of the numerical implementation by comparing our predictions with results published in Liu et al. (2016): (a) evolution of ratio $\dot{W}^{e} / \dot{W}^{p}$; (b) evolution of the macroscopic logarithmic strain components $E_{11}, E_{22}$ and $E_{33} ;$ (c) evolution of the macroscopic equivalent stress $\Sigma_{e q}$ normalized by the initial stress $\sigma_{0}$.

\subsubsection{Competition between void coalescence and macroscopic strain localization}

To start the analysis of the competition between void coalescence and macroscopic strain localization, three proportional stressing configurations are simulated. For these loadings, the Lode parameter $L$ is set to 1.0 and three values for the stress triaxiality ratio $T$ are considered: $0.8 ; 1.0$ and 2.0 . The matrix of the unit cell is assumed to be fully dense (hence, $f_{s 0}=0$ ) and the initial volume fraction of the primary void $f_{p 0}$ is set to 0.04. The evolution of some macroscopic quantities, relevant to study the competition between void coalescence and macroscopic strain localization, are plotted in Fig. 6. In unit cell computations, the equivalent strain $E_{e q}$ is often used as a measure of material ductility. Therefore, a critical equivalent strain, predicted by each indicator, has also been used to denote material failure. For comparison purposes, four symbols are marked in each curve of Fig. 6 to designate the level of the macroscopic equivalent strain corresponding to each criterion:

- A square $(\square)$ to designate $E_{e q}^{R}$ (when the reaction force component $\alpha_{3}$ of the dummy node reaches its maximum value).

- A circle (O) to designate $E_{e q}^{S}$ (when the equivalent stress $\Sigma_{e q}$ reaches its maximum value). 
- A star (*) to designate $E_{e q}^{B}$ (when the determinant of the acoustic tensor $\overrightarrow{\mathcal{N}} \cdot \mathcal{L} \cdot \overrightarrow{\mathcal{N}}$ vanishes).

- An up triangle $(\triangle)$ to designate $E_{e q}^{C}$ (when ratio $\dot{W}^{e} / \dot{W}^{p}$ reaches its minimum and negative value).

In view of the curves in Fig. 6, some conclusions can be drawn:

- For all of the stress triaxiality ratios considered, the critical equivalent strains predicted by the different criteria are classified as follows:

$$
E_{e q}^{S}<E_{e q}^{R}<E_{e q}^{B}<E_{e q}^{C} .
$$

- As expected, the different critical equivalent strains (namely, $E_{e q}^{S}, E_{e q}^{R}, E_{e q}^{B}$ and $E_{e q}^{C}$ ) decrease with the increase of stress triaxiality ratio $T$, reflecting the loss of ductility. Moreover, the dependency of the different critical equivalent strains on $T$ is more pronounced in the low stress triaxiality range and saturates as $T$ increases to a high level.

- The difference between the various critical equivalent strains decreases when the stress triaxiality ratio increases. For instance, the difference between $E_{e q}^{S}$ and $E_{e q}^{C}$ decreases from 0.73 for $T=0.8$ to 0.20 for $T=2.0$.

The effect of the stress triaxiality ratio on the onset of ductile failure will be further investigated in Section 5.3.3, where a wide range of $T$ will be considered and not only three particular values.

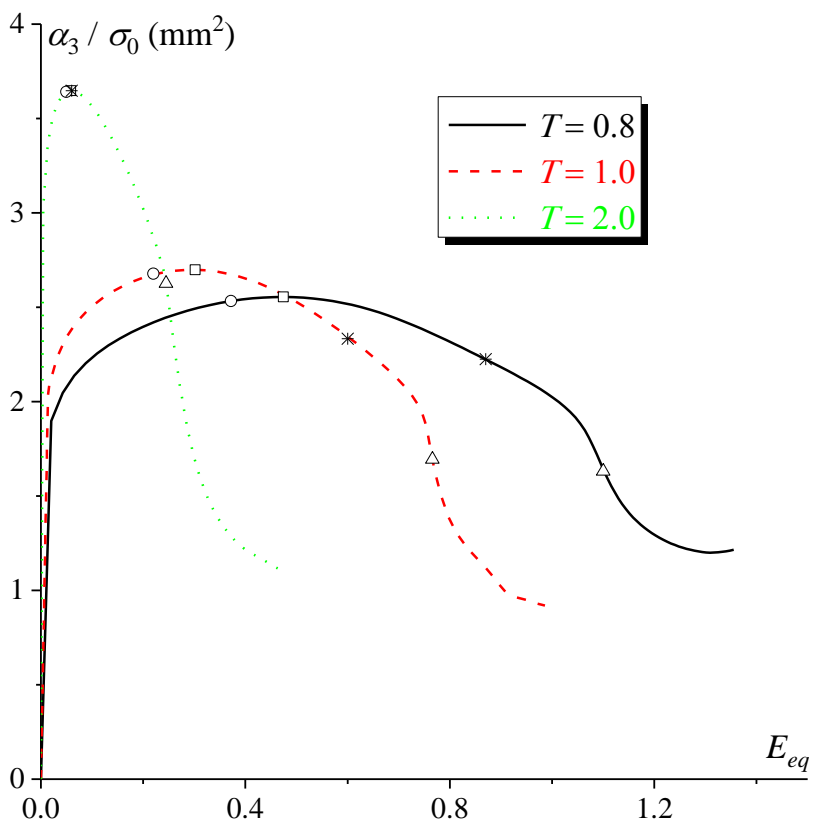

(a)

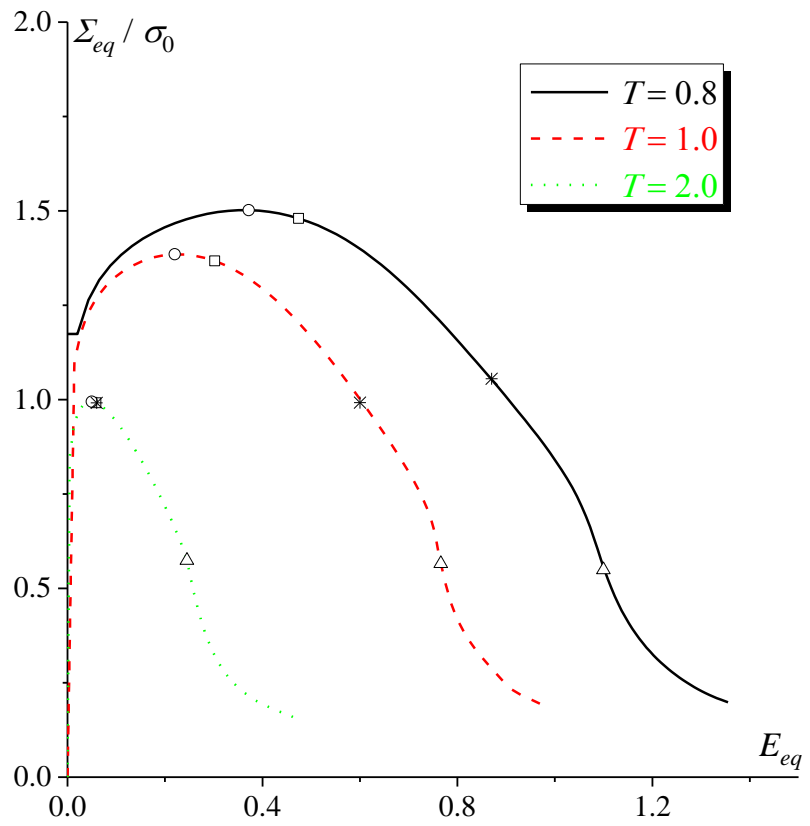

(b) 


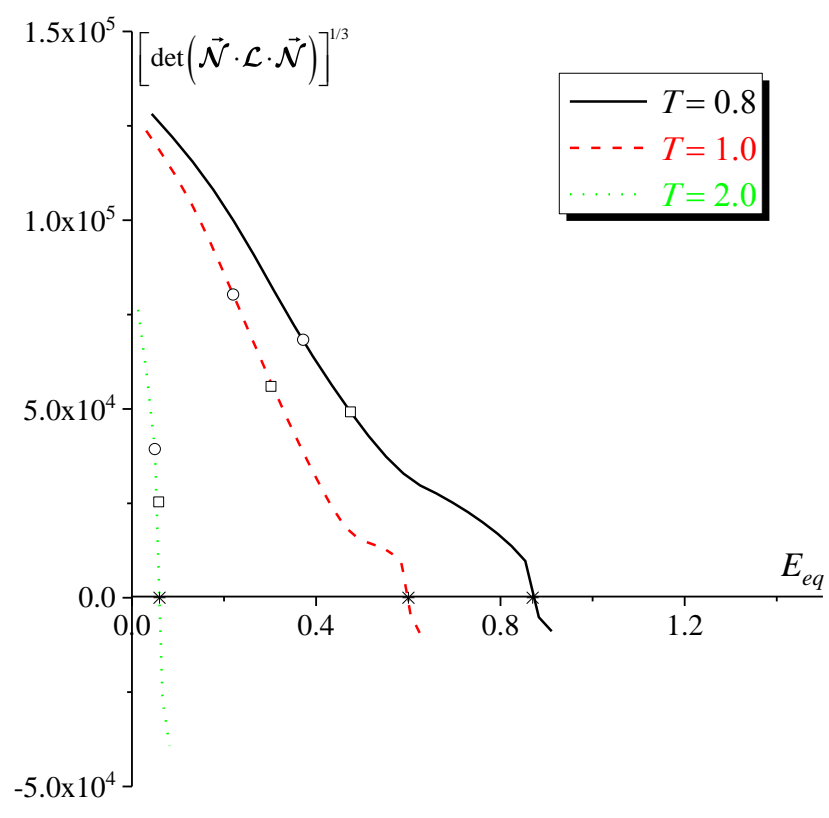

(c)

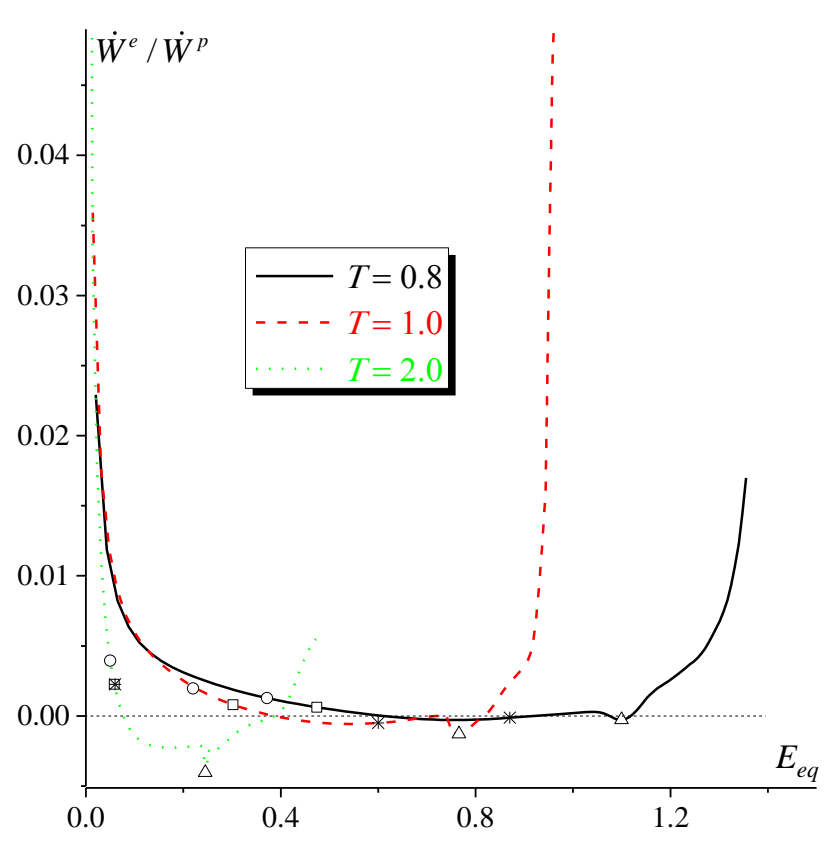

(d)

Fig. 6. Competition between void coalescence and macroscopic strain localization for proportional stressing configurations defined by $T=0.8 ; 1$ and 2 , with $L=1$ : (a) evolution of the reaction force component $\alpha_{3}$ of the dummy node normalized by the initial stress $\sigma_{0}$; (b) evolution of the equivalent stress $\Sigma_{e q}$ normalized by the initial stress $\sigma_{0}$;

(c) evolution of the cubic root of the determinant of the acoustic tensor $\overrightarrow{\mathcal{N}} \cdot \mathcal{L} \cdot \overrightarrow{\mathcal{N}} ;$ (d) evolution of ratio $\dot{W}^{e} / \dot{W}^{p}$.

\subsubsection{Effect of the stress triaxiality ratio on ductile failure}

An overview of the competition between void coalescence and strain localization is provided in Fig. 7, where the evolutions of the critical equivalent strains $E_{e q}^{S}, E_{e q}^{R}, E_{e q}^{B}$ and $E_{e q}^{C}$ are plotted against $T$ for the range $(0.7 \leq T \leq 3.0)$ and for three $L$ values $(-1 ; 0$ and 1.0). As one can see, all of the four limit strains decrease as $T$ increases for the different values of $L$. By examining the evolution of $E_{e q}^{C}$ and $E_{e q}^{B}$ for a given range of $T$, one can observe that $E_{e q}^{B}<E_{e q}^{C}$ regardless the value of $L$. Namely, localization bifurcation occurs before the attainment of void coalescence predicted by the energy-based criterion for the full range of $T$. For $T<2.0$, the limit strains $E_{e q}^{B}, E_{e q}^{R}$ and $E_{e q}^{S}$ are attained with $E_{e q}^{S}<E_{e q}^{R}<E_{e q}^{B}$, and the difference between these limit strains decreases as $T$ increases for the various values of $L$. For higher values of $T$, notably $T>2.0$, the curves corresponding to $E_{e q}^{B}, E_{e q}^{R}$ and $E_{e q}^{S}$ are almost indistinguishable. This result means that for high stress triaxiality levels, the maximum of the reaction force of the dummy node, the maximum equivalent stress and the bifurcation are reached at approximatively the same moment. This latter conclusion 
is fully consistent with the predictions obtained by Guo and Wong (2018), where it has been demonstrated that the maximum reaction force of the dummy node (used as strain localization criterion) and the maximum equivalent stress are simultaneously attained for high values of $T$ (typically higher than 1.5). On the other hand, the bifurcation criterion (used in the present contribution as a rigorous macroscopic strain localization criterion) appears to be less conservative than the criterion of maximum reaction force on the dummy node (used as strain localization criterion in Guo and Wong, 2018) for the range of low triaxialty $(T<2.0)$, and this is more remarkable for $L=1.0$. Whatever the adopted macroscopic strain localization criterion, defined as the maximum equivalent stress in Bomarito and Warner (2015) and Tekoğlu et al. (2015) or as the maximum reaction force in Guo and Wong (2018), the bifurcation criterion presented herein leads to relatively higher limit strains for $T<2.0$. For higher stress triaxiality $(2.0<T<3.0)$, all three strain localization criteria predict almost the same results.

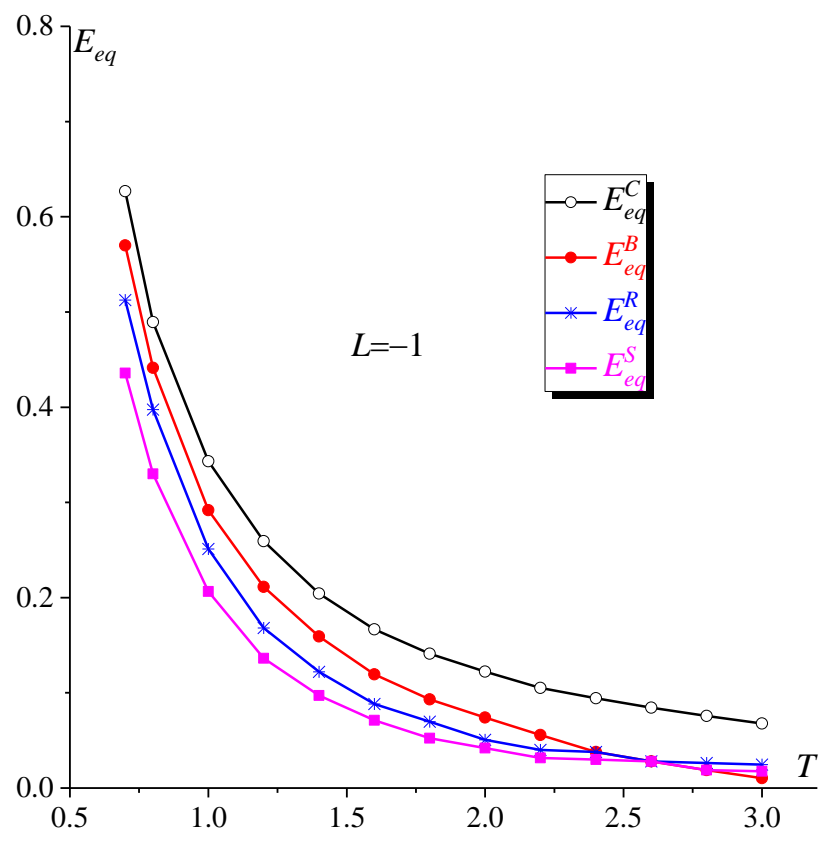

(a)

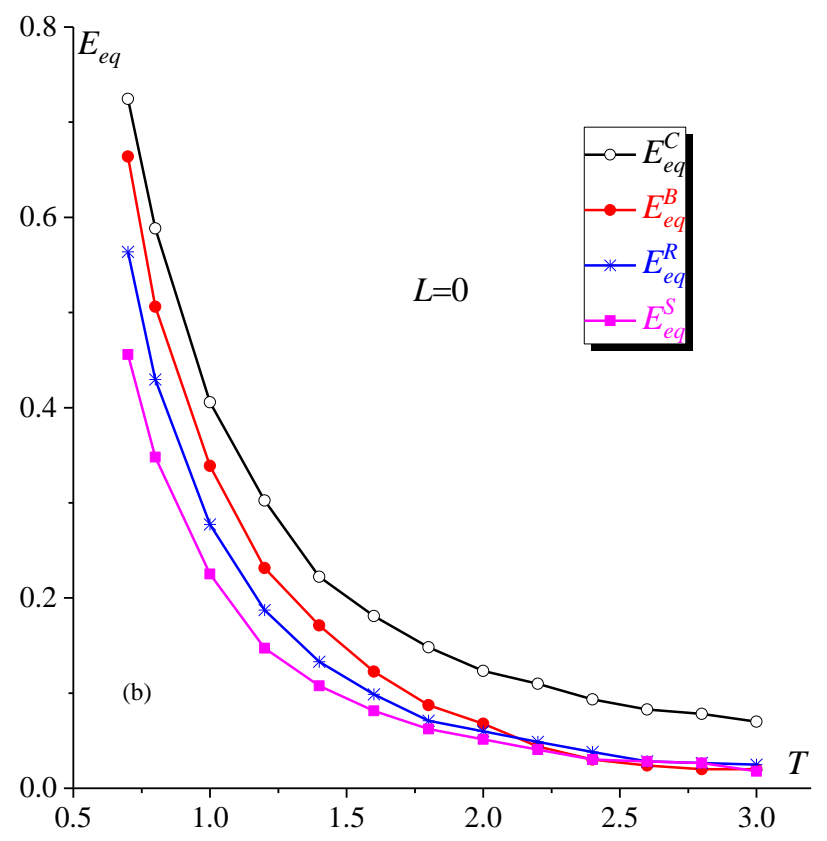

(b) 


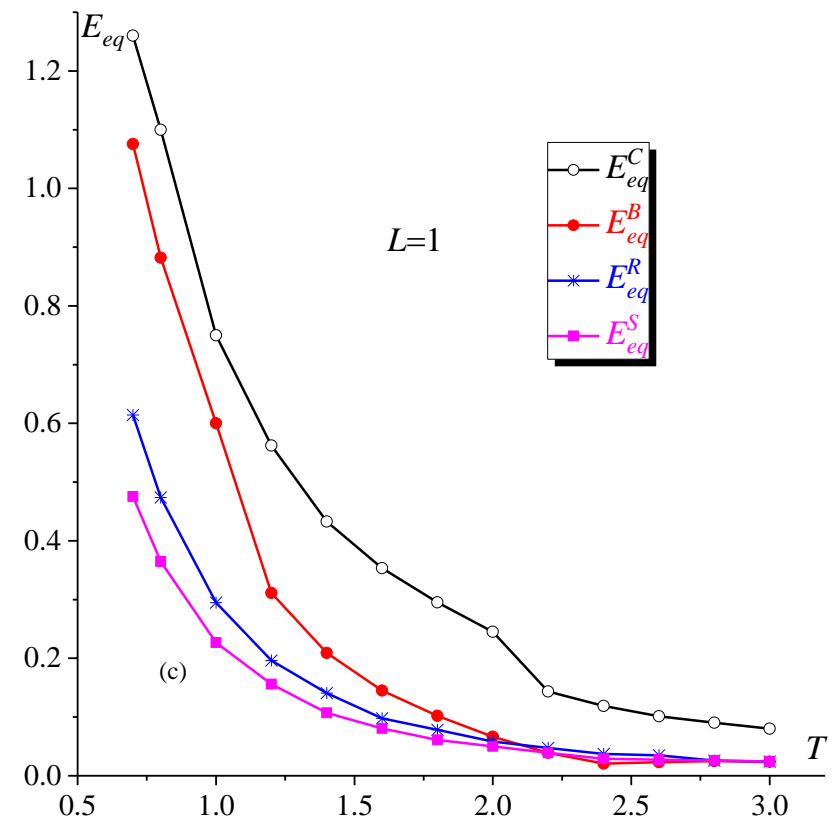

(c)

Fig. 7. Evolution of critical equivalent strains $E_{e q}^{S}, E_{e q}^{R}, E_{e q}^{B}$ and $E_{e q}^{C}$ over the range $0.7 \leq T \leq 3.0$ and for (a) $L=-1$; (b) $L=0 ;$ (c) $L=1$.

\subsubsection{Effect of the Lode parameter on ductile failure}

Special attention is now paid to the analysis of the effect of the Lode parameter $L$ on macroscopic strain localization and void coalescence. The evolutions of the critical equivalent strains $E_{e q}^{C}$ and $E_{e q}^{B}$ are plotted in Fig. 8 over the range $-1.0 \leq L \leq 1.0$ and for some particular $T$ values $(0.8 ; 1.2 ; 1.6 ; 2.0 ; 2.4$ and 3.0$)$. The loci of $E_{e q}^{C}$ and $E_{e q}^{B}$ are denoted by solid and dash lines, respectively. At first glance, it is clear that $E_{e q}^{C}$ is larger than $E_{e q}^{B}$ for the full range of $L$. For low to moderate levels of $T$, both $E_{e q}^{C}$ and $E_{e q}^{B}$ increase with $L$, especially for $0 \leq L \leq 1.0$, with different increase rates. Moreover, the difference between $E_{e q}^{C}$ and $E_{e q}^{B}$ increases with $L$ in the range $0 \leq L \leq 1.0$ for relatively low triaxiality levels. For high stress triaxiality levels (e.g., $T=2.4$ and $T=3.0), E_{e q}^{C}$ and $E_{e q}^{B}$ are quasi linear and almost constant for the full range of $L$. In other words, the effect of Lode parameter is more pronounced when the stress triaxiality is low. Regarding the effect of $L$, Barsoum and Faleskog (2011) and Dunand and Mohr (2014) have found that $E_{e q}^{C}$ performs a convex and non-symmetric function of $L(-1.0<L<1.0)$ by following the coalescence criterion developed in Needleman and Tvergaard (1992). This trend has been confirmed by Luo and Gao (2018) using a sandwiched unit cell model, but seems to be inconsistent with that observed in our investigation. This apparent 
inconsistency is explained by the convention adopted to determine $T$ and $L$ from $\beta_{1}$ and $\beta_{2}$ (see Section 3.1). In fact, several families of stress states $\left(\beta_{1}, \beta_{2}\right)$ could be obtained from a given $T$ and $L$, as demonstrated by Wong and Guo (2015). Consequently, for these different stress states, the predicted critical strains for macroscopic strain localization are quite distinct, and the same applies to void coalescence, although the stress triaxiality ratio $T$ and the Lode parameter $L$ remain the same. Therefore, with various stress states $\left(\beta_{1}, \beta_{2}\right)$, one may draw different conclusions regarding the effect of $L$ (see, e.g., Fig. 11 in Wong and Guo, 2015). With the convention for $L$ adopted in our investigation, we obtain trends that are similar to those observed in Zhu et al. (2018).

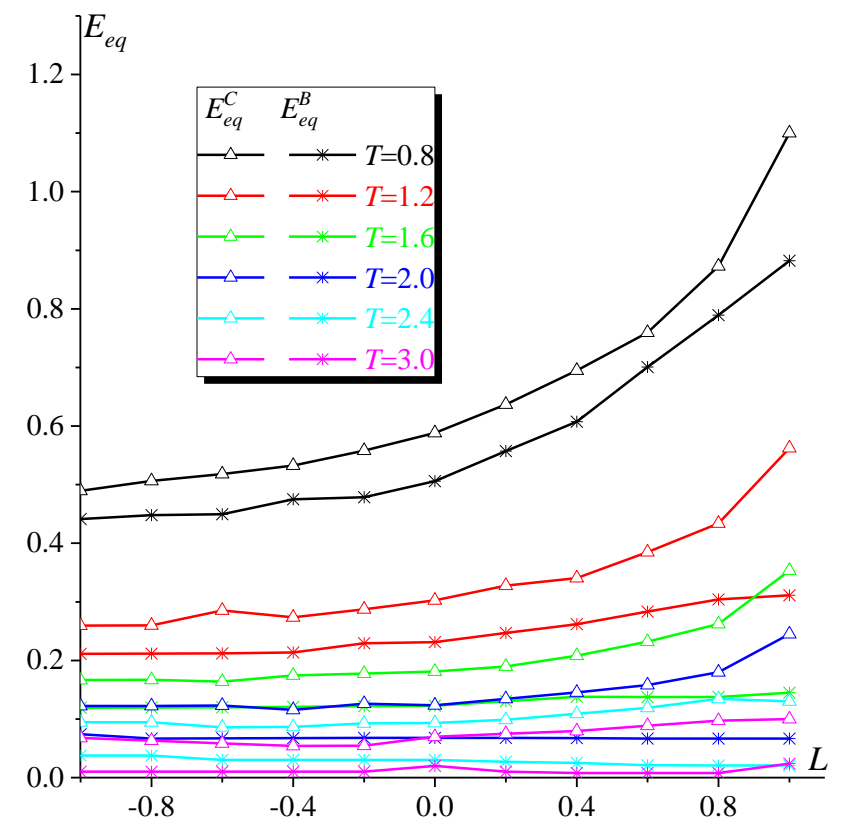

Fig. 8. Locus of limit strains $E_{e q}^{C}$ and $E_{e q}^{B}$ over the range $-1.0 \leq L \leq 1.0$ and for $T=0.8 ; 1.2 ; 1.6 ; 2.0 ; 2.4 ; 3.0$. 


\subsection{Proportional in-plane strain paths}

In the current section, the ductility limit of thin metal sheets is predicted and depicted through the conventional representation of forming limit diagrams (FLDs). For this purpose, we consider a unit cell subjected to proportional in-plane biaxial stretching along with plane-stress conditions in the out-of-plane direction (see Fig. 1b). We firstly demonstrate that void coalescence cannot be predicted by the energy-based criterion for this loading configuration (Section 5.4.1). The other indicators (namely, the criterion of maximum reaction force on the dummy node and the maximum equivalent stress criterion) are classically considered by the 'sheet metal forming' community as diffuse necking criteria and, hence, not relevant to predict FLDs. Consequently, the Rice bifurcation approach will be the only criterion used for predicting FLDs. Although this criterion has been widely used in the literatures to predict the onset of localized necking in thin metal sheets (Mansouri et al., 2014; Ben Bettaieb and Abed-Meraim, 2015; Akpama et al, 2017), this is the first time that it is coupled with 3D unit cell computations. The effects of two key factors on the ductility limits of thin metal sheets are investigated: the shape of the primary void (Section 5.4.2), and the presence of secondary population of voids (Section 5.4.3).

\subsubsection{Prediction of void coalescence by the energy-based criterion}

To check whether elastic unloading may occur during the application of proportional strain paths, the evolution of the strain component normal to the thickness direction $E_{33}$ is plotted in Fig. 9a versus the major in-plane strain $E_{11}$ for three representative strain-path ratios: $\rho=-0.5 ; 0.0 ; 1.0$. The minor in-plane strain $E_{22}$ is equal to $\rho E_{11}$. As one can see in Fig. 9a, $E_{33}$ decreases monotonically as the deformation proceeds. Hence, the deformation mode does not switch from elastoplastic loading to elastic unloading. Consequently, void coalescence is not predicted by the energy-based criterion. This observation is evidently related to, at least, two facts: the range of stress state associated with the applied strain paths, and the nature of the applied macroscopic loading. To thoroughly analyze the first fact, the evolution of the stress state during loading is plotted in the $(T, L)$-space for some representative strain-path ratios (Fig. 9b). It turns out from Fig. 9b that the stress triaxiality ratio $T$ does not exceed 0.7 for all the loading cases and, hence, the strain paths corresponding to the FLDs are not covered by the range of stress triaxiality studied in Section 5.3 (between 0.7 and 3.0). By making connection between Fig. 9b and the curves of Fig. 7, it appears immediately evident why the void coalescence, predicted by the energy-based criterion, is not reached for the strain paths considered here. To better illustrate this study, the evolution of the volume fraction of the primary void $f_{p}$, normalized by its initial value $f_{p 0}$, is plotted against the major strain $E_{11}$ in Fig. 9 c. For the strain-path ratio $\rho=-0.5$, ratio $f_{p} / f_{p 0}$ is almost constant during plastic deformation. This result can be explained by the very low stress 
triaxiality characterizing this particular strain path, as the stress state is near uniaxial tension state (Fig. 9b). In this case, only the void shape changes (from spherical to approximately ellipsoidal), without significant void growth. For the strain-path ratios $\rho=0.0$ and 1.0 , ratio $f_{p} / f_{p 0}$ increases slowly as plastic deformation proceeds. This slight evolution of void growth, without abrupt change, explains the difficulty in reaching void coalescence.

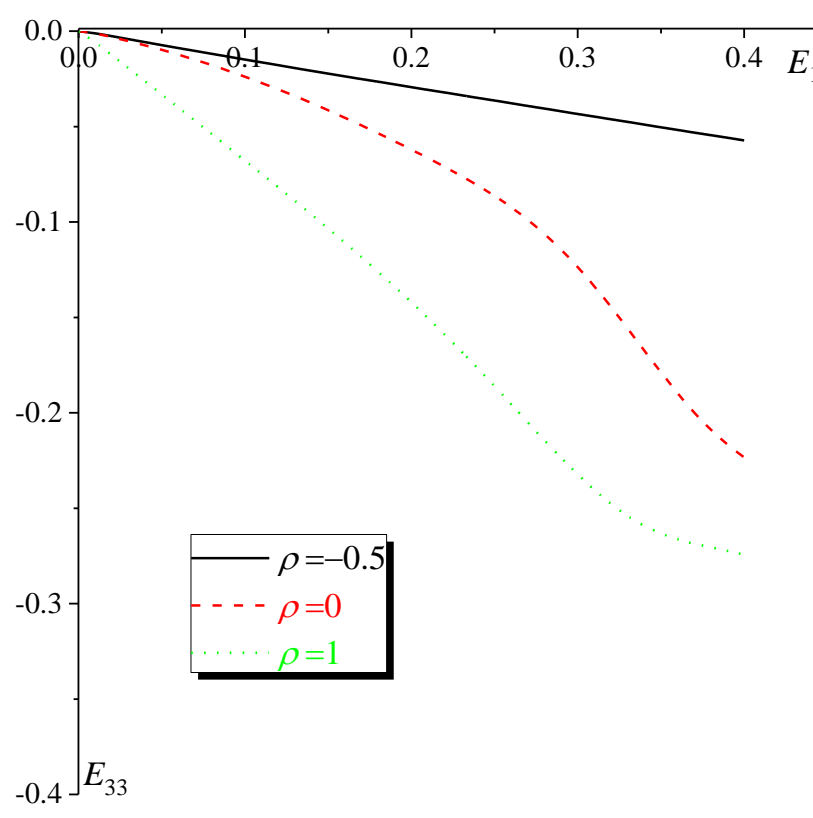

(a)

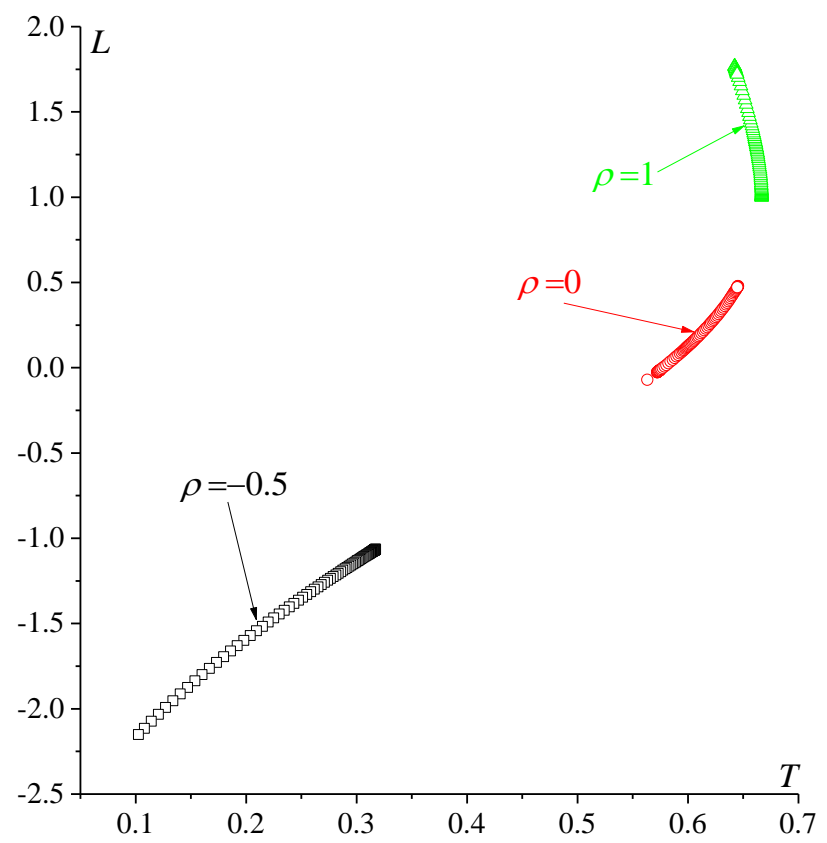

(b) 


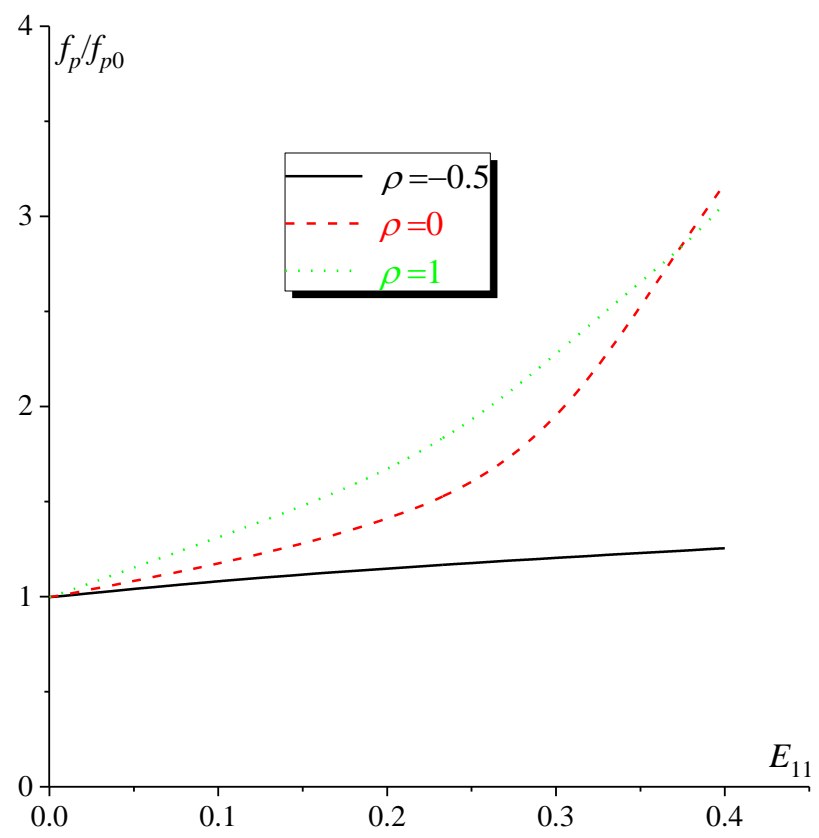

(c)

Fig. 9. Study for strain-path ratios $\rho=-0.5 ; 0.0 ; 1.0$ : (a) evolution of $E_{33}$ versus $E_{11}$; (b) distribution of the stress triaxiality ratio $T$ and the Lode parameter $L ;$ (c) volume fraction of the primary void $f_{p}$ normalized by $f_{p 0}$.

As to the nature of the applied macroscopic loading, for proportional in-plane strain paths, the strain components $E_{11}$ and $E_{22}$ are prescribed and are assumed to evolve monotonically during the loading. The strain component $E_{33}$ is determined by the plane-stress condition $\left(\Sigma_{33}=0\right)$, and this component generally decreases with plastic deformation. Consequently, macroscopic elastic unloading cannot occur in this case. By contrast, for proportional stressing, the stress ratios $\beta_{1}$ and $\beta_{2}$ hold constant and strain components $E_{11}, E_{22}$ and $E_{33}$ adjust to ensure that $\beta_{1}$ and $\beta_{2}$ keep their desired values. During loading, the instant when the sign of at least one of the components $\dot{E}_{11}, \dot{E}_{22}$ and $\dot{E}_{33}$ changes marks the onset of elastic unloading.

In several earlier contributions, void coalescence has been assumed to occur when the growth of the primary void exhibits an abrupt acceleration. As shown in Fig. 9c, a clear abrupt change in the void growth is not observed for any of the studied strain paths. This observation is due to the effect of neighboring unit cells on the deceleration of void growth. To elucidate this effect, let us consider two sheet configurations. In the first configuration, the sheet is defined by a 2D arrangement of voided unit cells, similar to the one shown in Fig. 1a, but with a single unit cell in the thickness of the sheet. As previously stated, void coalescence is not reached under this configuration for all of the applied strain paths. As to the second configuration (see Fig. 10), it is defined by a dense sheet crossed by a narrow voided band. The width ratio $\tilde{l}_{0} / l_{0}$ for the current simulations is set to 2.0 (see Fig. 10). This second configuration follows the same spirit as the initial imperfection approach 
introduced by Marciniak and Kuczynski (1967). Under this configuration, the sheet is subjected to the following displacement rate boundary conditions:

$$
\left\{\begin{array}{l}
\dot{U}_{1}=1 ; \\
\left.\dot{U}_{2}=0 \text { (plane strain in direction } 2\right) ; \\
\left.U_{3}=\text { free (plane stress in direction } 3\right) .
\end{array}\right.
$$

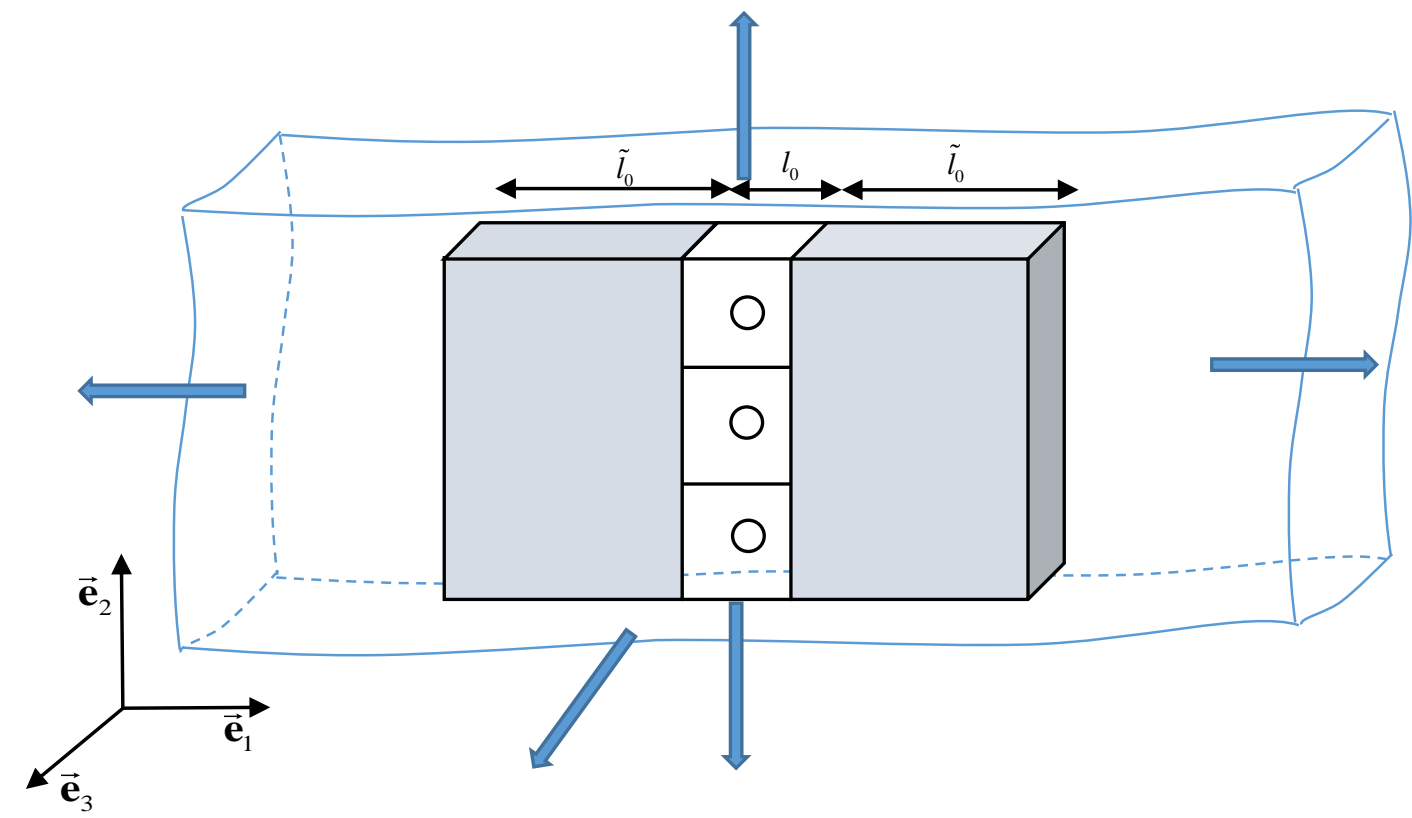

Fig. 10. Dense sheet crossed by a narrow voided band.

The application of the classical boundary conditions, given by Eq. (38), to the sheet configuration defined in Fig. 10 leads to localization of the deformation inside the voided band, along with relatively slight deformation followed by elastic unloading outside the band (called safe zone), as shown in Fig. 11a. In this figure, $E_{33}^{\text {Band }}$, $E_{33}^{\text {Safe }}$ and $E_{33}^{\text {Classic }}$ refer to the 33 component of the macroscopic strain inside the band, in the safe zone and as obtained by classic unit cell computations (configuration 1), respectively. For configuration 2, a rapid void growth can be observed, as displayed in Fig. 11b, which may promote the onset of void coalescence. Consequently, void coalescence is dependent on the stress state inside the unit cell and also on the mechanical behavior of regions surrounding this unit cell. The same trends are obtained for the other strain paths, but to be concise, the corresponding results are not presented here. If the width ratio $\tilde{l}_{0} / l_{0}$ is chosen to be very large (a very small band width), an abrupt change in void growth can be readily obtained, thus characterizing the onset of void coalescence. It shall be noted that the configuration presented in Fig. 10 has been widely used in the literature to predict the onset of void coalescence and strain localization in voided solids (Tekoğlu et al., 2015; Luo and Gao, 2018). 


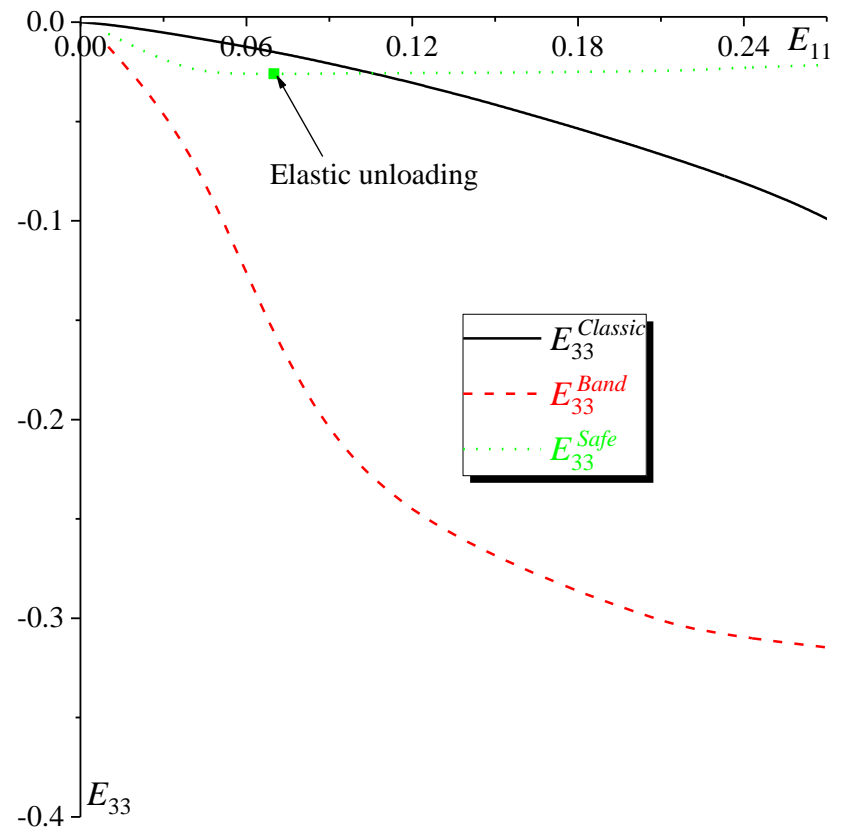

(a)

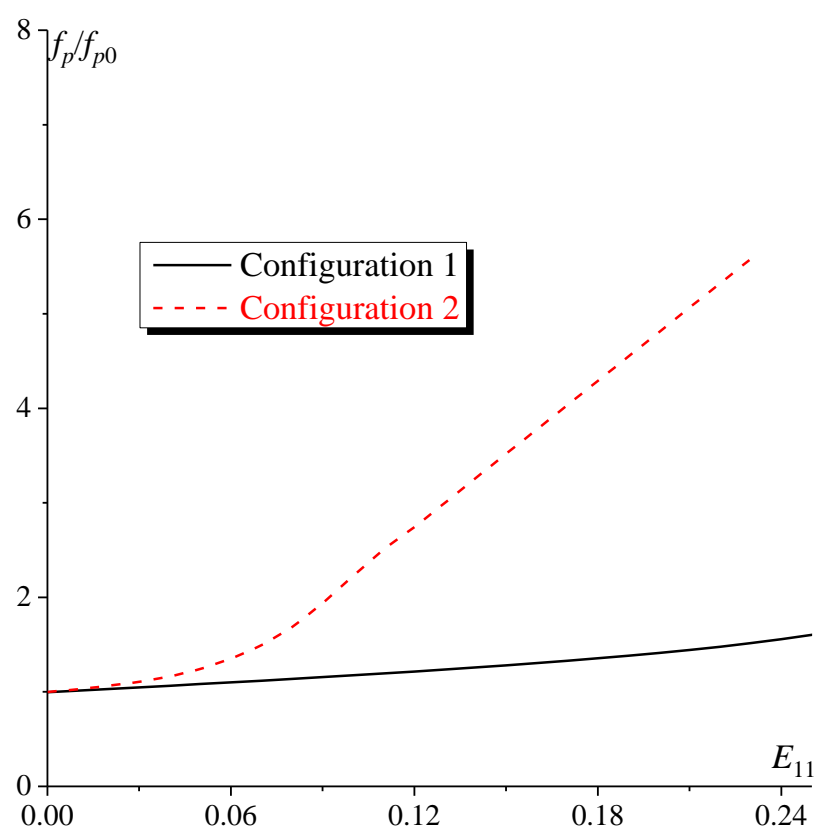

(b)

Fig. 11. Effect of the neighborhood of the unit cell on: (a) the evolution of the strain component in the thickness direction outside the band; (b) the growth of the primary void.

\subsubsection{Effect of initial primary void shape on the ductility limits of thin sheets}

In the previous sections, the initial shape of the primary void was assumed to be spherical. However, some experimental evidences show that voids are often non-spherical in actual materials, such as rolled plates (Gao and Kim, 2006). And even for materials containing initially spherical voids, the void shape is likely to change as a function of the applied stress state. Voids in real materials may have, for example, the shape of long, prolate ellipsoids, if they nucleate around segregations previously elongated by a rolling process; at the opposite extreme, they may look like wide, oblate ellipsoids if they happen to grow from cleavage cracks generated in the hard phase of a dual-phase structure (see, e.g., Pineau and Joly, 1991). There is thus a need for accurately modeling the effect of void shape on the occurrence of ductile failure. In this aim, the Gurson model has been extended by Gologanu et al. $(1993,1994)$ to study the growth of non-spherical voids embedded in a ductile dense matrix. In the present contribution, the developed approach is used for the investigation of this effect on the shape and the level of forming limit diagrams. To this end, three shapes are considered for the primary void: oblate, spherical and prolate. Oblate and prolate voids are assumed to be axisymmetric about 2-axis, and their initial aspect ratio is defined as $\mathcal{W}_{0}=r_{p 2} / r_{p 1}=r_{p 2} / r_{p 3}$. Thus, $\mathcal{W}_{0}=1$ corresponds to a spherical void (Fig. 12b), $\mathcal{W}_{0}=1 / 3$ to oblate void (Fig. 12a), and $\mathcal{W}_{0}=3$ to prolate void (Fig. 12c). In these 
three cases, the initial volume fraction of the primary void is taken to be $f_{p 0}=0.04$. The metal matrix is assumed to be fully dense (hence, $f_{s 0}=0.0$ ).

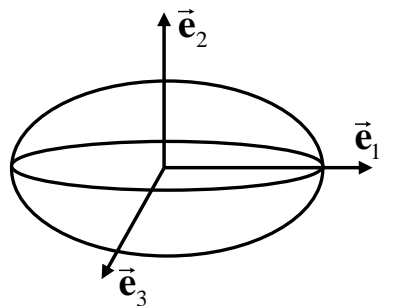

(a)

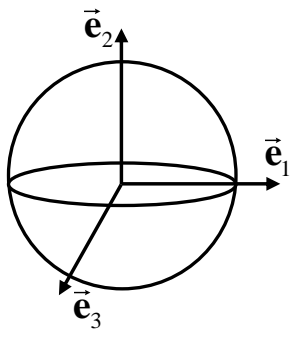

(b)

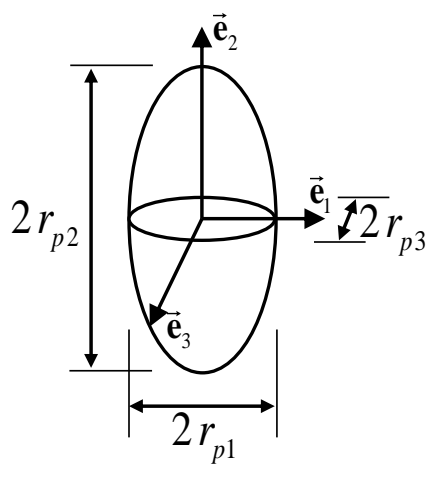

(c)

Fig. 12. Geometric representation of three void shapes: (a) oblate void $\left(\mathcal{W}_{0}=1 / 3\right)$; (b) spherical void; (c) prolate void

$$
\left(\mathcal{W}_{0}=3\right) \text {. }
$$

The effect of void aspect ratio on the void-induced softening phenomenon has been highlighted in several earlier investigations (Pardoen and Hutchinson, 2000; Keralavarma and Benzerga, 2010). In the present section, attention is focused on the effect of the initial void shape on the ductility limits. As clearly shown in Fig. 13a, the initial void shape slightly affects the left hand side of the FLDs. More interestingly, the ductility limit increases as the aspect ratio $\mathcal{W}_{0}$ decreases in the range of positive strain-path ratios. This result is quite expectable considering the fact that the void growth mechanism should be more important for the unit cell with $\mathcal{W}_{0}=3$ and, consequently, the ductility of the unit cell is negatively affected by this void growth. The predictions of Fig. 13a are fully consistent with the results published in Pardoen and Hutchinson (2000) and in Keralavarma and Benzerga (2010). To further analyze this effect, the initial macroscopic yield loci corresponding to the different unit cell configurations are plotted in Fig. 13b. Focus is confined to the first quadrant of these yield loci ( $\Sigma_{11} \geq 0$ and $\Sigma_{22} \geq 0$ ), as the stress states corresponding to the whole range of strain paths are located in this first quadrant (from uniaxial to equibiaxial tension state). In the case of nonspherical shape (e.g., ellipsoidal) for the primary void, there exists a preferred material orientation and the macroscopic plastic behavior reveals to be anisotropic (even if the metal matrix is elastically and plastically isotropic). Consequently, the change in the initial aspect ratio $\mathcal{W}_{0}$ would induce a change in the curvature of the macroscopic yield locus, as shown in Fig. 13b. This curvature has an important impact on the predicted ductility limits, especially near the equibiaxial tension state (Barlat, 1987): the sharper the yield surface, the lower the corresponding ductility limit. 


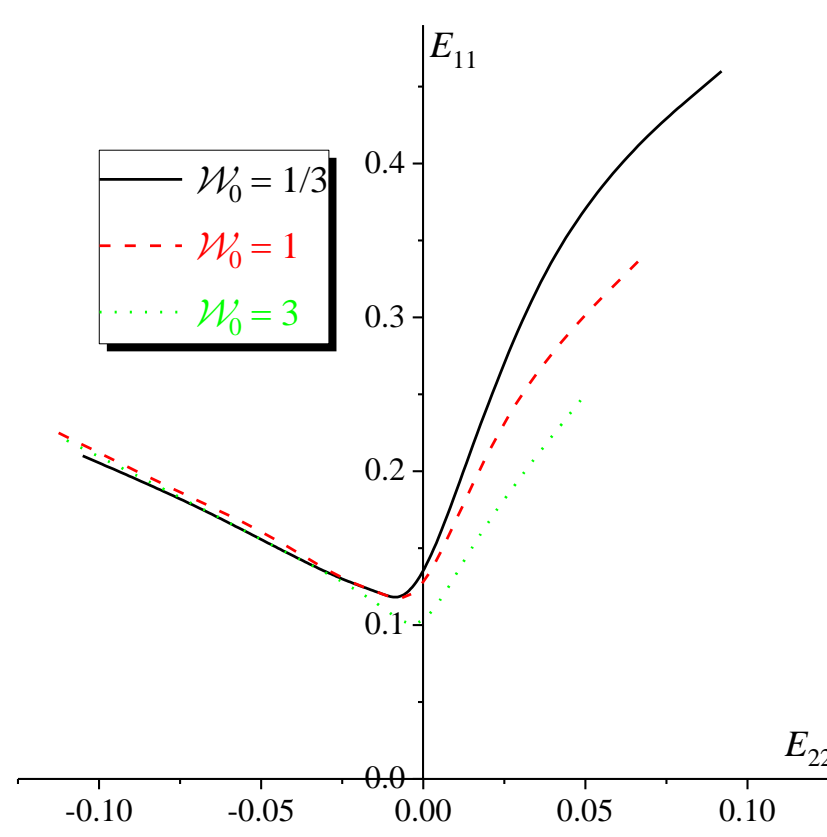

(a)

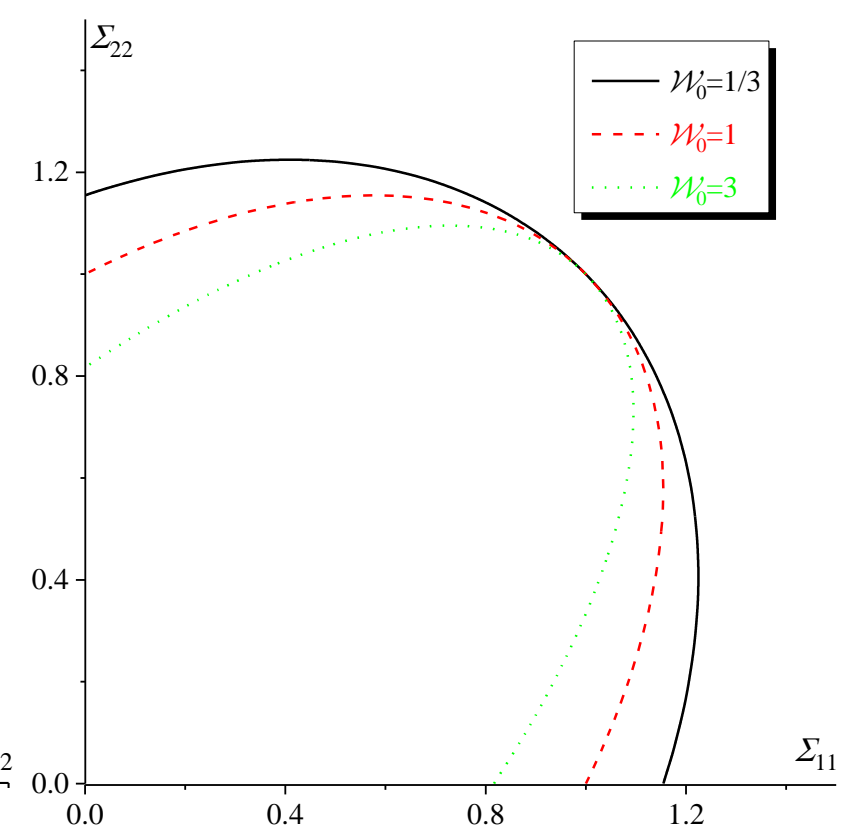

(b)

Fig. 13. Effect of initial shape of the primary void on: (a) the predicted forming limit diagrams; (b) the shape of the macroscopic yield loci for the quadrant $\left(\Sigma_{11} \geq 0\right.$ and $\left.\Sigma_{22} \geq 0\right)$.

\subsubsection{Effect of secondary population of voids on the ductility limits of thin sheets}

Just far, we have consistently assumed that the metal matrix is fully dense. However, several experimental observations point out the potential presence of secondary (small) void populations, in addition to the primary void, in most engineering materials. Gao and Kim (2006) and Fabrègue and Pardoen (2008) have developed two extensions of the Gurson model to take into account the impact of secondary void population on the material behavior. It turns out from these investigations that nucleation and growth of secondary voids accelerate the coalescence of the primary void and, thus, lead to a reduction of material ductility. In the present contribution, this effect is investigated by using the Gurson constitutive framework for the modeling of the mechanical behavior of the matrix, as stated in Appendix A. The secondary voids are assumed to be uniformly distributed over the metal matrix and three distinct values for their initial volume fraction $f_{s 0}$ are considered: 0.0 (which corresponds to a fully dense matrix), 0.01 and 0.04 . The values of $f_{s 0}$ adopted here are for the only purpose of demonstrating the effect of secondary voids on void coalescence and macroscopic strain localization. No attempt is made to represent actual physical values. As depicted in Fig. 14a, the initial volume fraction of secondary voids $f_{s 0}$ affects the limit strains. In the range of positive strain-path ratios, the ductility limits decrease as $f_{s 0}$ increases. However, in the range of negative strain-path ratios, the forming limit curves are less sensitive to the value of $f_{s 0}$. This result is quite expectable considering the fact that the growth of 
primary as well as secondary voids increases with the strain-path ratio. To further investigate the effect of the secondary void population on the mechanical behavior, the evolution of the microscopic equivalent stress $\sigma_{e q}$ in a finite element near the primary void is plotted for the extreme strain-path ratios $(\rho=-0.5$ and $\rho=1.0)$. As one can see in Fig. 14b, the stress plots corresponding to $\rho=1.0$ are more sensitive to the value of $f_{s 0}$. In this case, the microscopic equivalent stress reaches its maximum earlier and decreases more rapidly for the case $f_{s 0}=0.04$. By contrast, for $\rho=-0.5$, the three stress plots are rather similar, and local softening is not observed in this case. This analysis may further explain the effect of $f_{s 0}$ on the predicted FLDs.

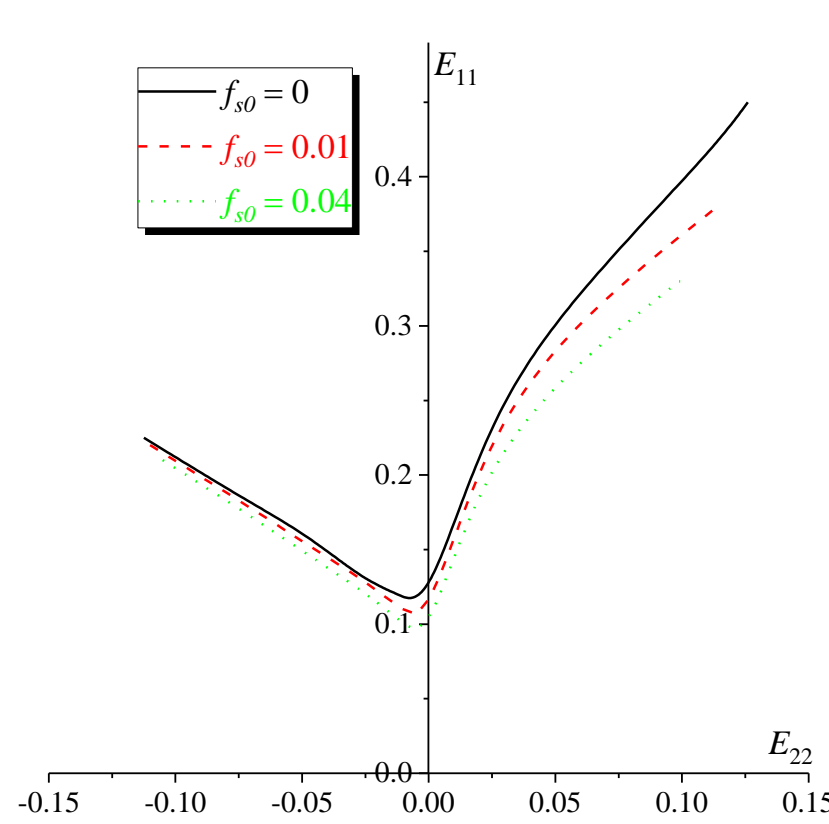

(a)

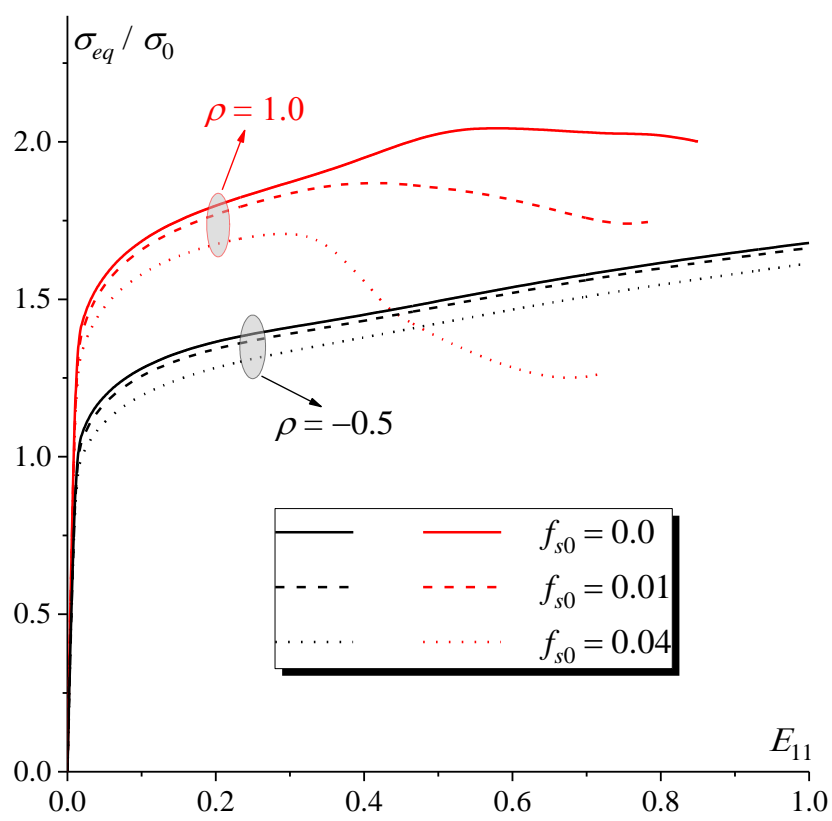

(b)

Fig. 14. Effect of the initial volume fraction of secondary voids on: (a) the predicted forming limit diagrams; (b) the evolution of the microscopic equivalent stress $\sigma_{e q}$ normalized by the initial yield stress $\sigma_{0}$.

\section{Concluding remarks}

In the present contribution, the Rice bifurcation approach has been coupled with the periodic homogenization scheme to predict the onset of macroscopic strain localization in 3D voided materials. For proportional stressing, the predictions given by the Rice bifurcation approach have been compared with those obtained by a void coalescence criterion and other strain localization indicators established in the scientific literature. This comparative study suggests that strain localization predicted by the Rice bifurcation approach acts as a precursor to void coalescence predicted by an elastic unloading criterion, when the stress triaxiality ratio $T$ ranges between 0.7 and 3.0. On the other hand, when $T \leq 2$, the limit strains predicted by the Rice bifurcation 
theory are much larger than those predicted by other localization criteria defined in the literature (Bomarito and Warner, 2015; Tekoğlu et al., 2015 and Guo and Wong, 2018). However, for $T>2$, the different strain localization criteria predict almost the same limit strains. The effect of the Lode parameter on the limit strains predicted by the different criteria has also been investigated, and we have demonstrated that this effect is more important for low values of $T$. The developed approach has also been applied to predict the forming limit diagrams of voided thin metal sheets. We have shown, through numerical investigations, that the ductility of thin metal sheets is only limited by the onset of strain localization, as void coalescence is not observed for the considered range of strain paths. To assess its reliability, the developed approach has been used for examining the effect of two key physical factors on the prediction of forming limit diagrams: the initial shape of primary void, and the presence of secondary void population. It has been pointed out from this analysis that the initial shape of primary void induces initial anisotropy in the macroscopic behavior of the unit cell and, hence, its effect is more pronounced in the range of the positive strain-path ratios. As to the presence of secondary void population, the initial volume fraction of these secondary voids has a negative impact on the ductility limit of the unit cell, especially in the right hand side of the FLD. This study would be completed by analyzing the competition between microscopic (local) and macroscopic strain localization. This will be one of the objectives of future applications of the developed approach.

\section{Acknowledgments}

The first author is grateful to the China Scholarship Council for providing him a $\mathrm{PhD}$ grant during the preparation of this work.

\section{Appendix A. Constitutive model for the metal matrix}

To account for the effect of secondary population of voids, the mechanical behavior of the metal matrix is modeled by the original Gurson porous model (Gurson, 1977). The same methodology can be applied for any other constitutive framework (for instance, the GTN model to include void nucleation and coalescence). A typical finite element mesh for the unit cell is presented in Fig. A. 1.

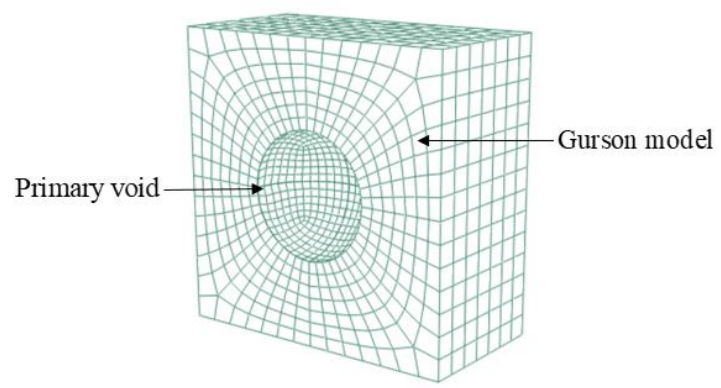

Fig. A. 1. Finite element mesh for one-half unit cell, for illustration. 
At the microscopic level, a relationship similar to Eq. (4) can be derived by combining the constitutive relations of the metal matrix:

$$
\dot{\mathbf{n}}=\ell: \mathbf{g}
$$

The expression of the microscopic analytical tangent modulus $\ell$ is determined from the following developments. As a departure point for these developments, the microscopic velocity gradient $\mathbf{g}$ is additively decomposed into the strain rate $\mathbf{d}$ and the spin tensor $\mathbf{w}$ :

$$
\mathbf{g}=\mathbf{d}+\mathbf{w} \quad ; \quad \mathbf{d}=\frac{1}{2}\left(\mathbf{g}+\mathbf{g}^{T}\right) \quad ; \quad \mathbf{w}=\frac{1}{2}\left(\mathbf{g}-\mathbf{g}^{T}\right)
$$

The strain rate $\mathbf{d}$ is itself split into its elastic and plastic parts $\mathbf{d}^{e}$ and $\mathbf{d}^{p}$ :

$$
\mathbf{d}=\mathbf{d}^{e}+\mathbf{d}^{p} .
$$

In a co-rotational material frame (we adopt here the co-rotational frame associated with the Jaumann objective rate), the Cauchy stress rate is described with the following hypoelastic law:

$$
\dot{\boldsymbol{\sigma}}=\mathbf{c}^{e}: \mathbf{d}^{e},
$$

where $\mathbf{c}^{e}$ is the fourth-order elasticity tensor.

The plastic strain rate $\mathbf{d}^{p}$ is determined by the normality rule:

$$
\mathbf{d}^{p}=\dot{\lambda} \frac{\partial \Phi}{\partial \boldsymbol{\sigma}}
$$

with $\dot{\lambda}$ denoting the plastic multiplier, and $\Phi$ the Gurson yield function defined as:

$$
\Phi=\left(\frac{\sigma_{e q}}{\sigma_{y}}\right)^{2}+2 f_{s} \cosh \left(\frac{3 \sigma_{h}}{2 \sigma_{y}}\right)-\left(1+f_{s}^{2}\right) \leq 0,
$$

where

- $\sigma_{e q}$ is the von Mises equivalent stress, equal to $\left(3 \boldsymbol{\sigma}_{d e v}: \boldsymbol{\sigma}_{d e v} / 2\right)^{1 / 2}$.

- $\boldsymbol{\sigma}_{d e v}$ and $\sigma_{h}=\operatorname{tr}(\boldsymbol{\sigma}) / 3$ are the deviatoric and hydrostatic parts of the Cauchy stress tensor $\boldsymbol{\sigma}$, respectively.

- $\sigma_{y}$ is the yield stress of the dense metal matrix, defined by the Swift hardening law:

$$
\sigma_{y}=K\left(\varepsilon_{0}+\bar{\varepsilon}^{p}\right)^{n}
$$

where $K, \varepsilon_{0}$ and $n$ are hardening parameters, and $\bar{\varepsilon}^{p}$ is the equivalent plastic strain of the dense metal matrix. 
Note that setting $f_{s}=0$, one recovers the conventional $J_{2}$ flow theory with isotropic hardening. This particular case will be used when the metal matrix is assumed to be fully dense.

In the current investigation, only growth of secondary voids is considered (i.e., the effects of nucleation of new secondary voids and coalescence between existing voids are neglected). By neglecting the elastic volume change, the rate of the secondary void volume fraction reads:

$$
\dot{f}_{s}=\left(1-f_{s}\right) \operatorname{tr}\left(\mathbf{d}^{p}\right) \text {. }
$$

The equivalent plastic strain rate $\dot{\bar{\varepsilon}}^{p}$ is obtained from the equivalence principle in terms of rate of plastic work for the metal matrix and its dense part:

$$
\left(1-f_{s}\right) \sigma_{y} \dot{\bar{\varepsilon}}^{p}=\boldsymbol{\sigma}: \mathbf{d}^{p} \Leftrightarrow \dot{\bar{\varepsilon}}^{p}=\frac{\boldsymbol{\sigma}: \mathbf{d}^{p}}{\left(1-f_{s}\right) \sigma_{y}} .
$$

The activation of plastic flow is governed by the application of the Kuhn-Tucker constraints:

$$
\begin{aligned}
& \text { elastic loading / unloading: } \Phi \leq 0 ; \dot{\Phi}<0 ; \dot{\lambda}=0 ; \\
& \text { elastoplastic loading: } \Phi=0 ; \dot{\Phi}=0 ; \quad \dot{\lambda}>0 .
\end{aligned}
$$

In the case of elastoplastic loading, condition $\dot{\Phi}=0$ can be expanded as follows:

$$
\dot{\Phi}=\mathbf{V}_{\boldsymbol{\sigma}}: \dot{\boldsymbol{\sigma}}+V_{\sigma_{y}} \dot{\sigma}_{y}+V_{f_{s}} \dot{f}_{s}=0,
$$

where:

$$
\begin{aligned}
& \mathbf{V}_{\boldsymbol{\sigma}}=\frac{\partial \Phi}{\partial \boldsymbol{\sigma}}=\frac{1}{\sigma_{y}}\left[3 \frac{\boldsymbol{\sigma}_{d e v}}{\sigma_{y}}+f \cosh \left(\frac{3 \sigma_{h}}{2 \sigma_{y}}\right) \mathbf{I}_{2}\right] \\
& V_{\sigma_{y}}=\frac{\partial \Phi}{\partial \sigma_{y}}=-\frac{1}{\sigma_{y}}\left[2\left(\frac{\sigma_{e q}}{\sigma_{y}}\right)^{2}+3 f \sinh \left(\frac{3 \sigma_{h}}{2 \sigma_{y}}\right)\right] ; \\
& V_{f}=\frac{\partial \Phi}{\partial f}=2 \cosh \left(\frac{3 \sigma_{h}}{2 \sigma_{y}}\right)-2 f .
\end{aligned}
$$

The substitution of Eqs. (A.3), (A.5) and (A.12) into Eq. (A.4) leads to the following expression for $\dot{\boldsymbol{\sigma}}$ :

$$
\dot{\boldsymbol{\sigma}}=\mathbf{c}^{e}:\left(\mathbf{d}-\mathbf{d}^{p}\right)=\mathbf{c}^{e}:\left(\mathbf{d}-\dot{\lambda} \mathbf{V}_{\boldsymbol{\sigma}}\right)=\mathbf{c}^{e p}: \mathbf{d} .
$$

The combination of the above equations allows us to obtain the following expression for the plastic multiplier $\dot{\lambda}:$

$$
\dot{\lambda}=\frac{\mathbf{V}_{\boldsymbol{\sigma}}: \mathbf{c}^{e}: \mathbf{d}}{H_{\lambda}} \quad \text { where } \quad H_{\lambda}=\mathbf{V}_{\boldsymbol{\sigma}}: \mathbf{c}^{e}: \mathbf{V}_{\boldsymbol{\sigma}}-\frac{V_{\sigma_{y}}}{(1-f)} \frac{\left(\boldsymbol{\sigma}: \mathbf{V}_{\boldsymbol{\sigma}}\right)}{\sigma_{y}} \frac{\partial \sigma_{y}}{\partial \bar{\varepsilon}^{p}}
$$


The expression of the elastoplastic tangent modulus $\mathbf{c}^{e p}$ can be derived by combining Eqs. (A.13) and (A.14):

$$
\mathbf{c}^{e p}=\mathbf{c}^{e}-\alpha \frac{\left(\mathbf{c}^{e}: \mathbf{V}_{\boldsymbol{\sigma}}\right) \otimes\left(\mathbf{V}_{\boldsymbol{\sigma}}: \mathbf{c}^{e}\right)}{H_{\lambda}},
$$

where $\alpha=0$ for elastic loading or unloading, and $\alpha=1$ for elastoplastic loading.

As demonstrated by Mansouri et al. (2014), the microscopic tangent modulus $\boldsymbol{\ell}$ is related to the elastoplastic tangent modulus $\mathbf{c}^{e p}$ by the following relationship:

$$
\ell=\mathbf{c}^{e p}+\ell^{1}-\ell^{2}-\ell^{3}
$$

where $\ell^{1}, \ell^{2}$ and $\ell^{3}$ are fourth-rank tensors that originate from the large strain framework and which are given by the following index forms:

$$
\forall i, j, k, l=1,2,3: \quad \ell_{i j k l}^{1}=\sigma_{i j} \delta_{k l} \quad ; \quad \ell_{i j k l}^{2}=\frac{1}{2}\left(\sigma_{j l} \delta_{i k}+\sigma_{j k} \delta_{i l}\right) \quad ; \quad \ell_{i j k l}^{3}=\frac{1}{2}\left(\sigma_{i k} \delta_{j l}-\sigma_{i l} \delta_{j k}\right) .
$$

\section{Appendix B. Periodic boundary conditions}

We detail in the following developments (i.e., point-by-point) how the fully PBCs are practically applied on the opposite faces $B_{1}^{-}$and $B_{1}^{+}$, defined by the coordinates $x_{01}=-l_{0} / 2$ and $x_{01}=l_{0} / 2$, respectively. Considering two nodes $M^{-}$and $M^{+}$, with identical coordinates in 2 and 3 directions on faces $B_{1}^{-}$and $B_{1}^{+}$ (Fig. B. 1), the velocity of these two nodes can be determined from Eq. (2):

$$
\dot{\mathbf{x}}_{M^{-}}=\mathbf{G} \cdot \mathbf{x}_{M^{-}}+\mathbf{v}_{M^{-}}^{p e r} \quad ; \quad \dot{\mathbf{x}}_{M^{+}}=\mathbf{G} \cdot \mathbf{x}_{M^{+}}+\mathbf{v}_{M^{+}}^{p e r}
$$

The periodic boundary conditions imply that $\mathbf{v}_{M^{-}}^{\text {per }}=\mathbf{v}_{M^{+}}^{p e r}$. Consequently, integration of the combination of Eqs. (B.1) 1 and (B.1) yields:

$$
\mathbf{x}_{M^{+}}-\mathbf{x}_{M^{-}}=\mathbf{F} \cdot\left(\mathbf{x}_{0 M^{+}}-\mathbf{x}_{0 M^{-}}\right)
$$

where $\mathbf{F}$ is the macroscopic deformation gradient, with $F_{i j}$ its $i j^{\text {th }}$ component. $\mathbf{F}$ is related to the macroscopic velocity gradient $\mathbf{G}$ by the following relationship (Ben Bettaieb et al., 2012):

$$
\mathbf{G}=\dot{\mathbf{F}} \cdot \mathbf{F}^{-1} .
$$

On the other hand, the difference $\mathbf{x}_{M^{+}}-\mathbf{x}_{M^{-}}$is defined as follows:

$$
\mathbf{x}_{M^{+}}-\mathbf{x}_{M^{-}}=\left(\mathbf{u}_{M^{+}}-\mathbf{u}_{M^{-}}\right)+\left(\mathbf{x}_{0 M^{+}}-\mathbf{x}_{0 M^{-}}\right),
$$


where $\mathbf{u}_{M^{+}}$and $\mathbf{u}_{M^{-}}$are the displacements of nodes $M^{+}$and $M^{-}$, respectively. The comparison of Eq. (B.2) and Eq. (B.4) leads to the following relationship between the displacements $\mathbf{u}_{M^{+}}$and $\mathbf{u}_{M^{-}}$:

$$
\mathbf{u}_{M^{+}}-\mathbf{u}_{M^{-}}=\left(\mathbf{F}-\mathbf{I}_{2}\right) \cdot\left(\mathbf{x}_{0 M^{+}}-\mathbf{x}_{0 M^{-}}\right)=\left(\mathbf{F}-\mathbf{I}_{2}\right) \cdot\left[\begin{array}{l}
l_{0} \\
0 \\
0
\end{array}\right] \text {. }
$$

Practically, the constraints summarized by Eq. (B.5) are applied by using the multi-point constraints (MPC) option of ABAQUS for each node pair $\left(M^{-}, M^{+}\right)$. The Homtools enables to automatically identify all the node pairs of faces $B_{1}^{-}$and $B_{1}^{+}$, and to apply the MPC between the nodes from the same node pair. To easily manage the application of the macroscopic loading and, more interestingly, to automatize the determination of the macroscopic mechanical response, a reference point (using the terminology of ABAQUS), designated $R P_{1}$, is created. The macroscopic loading is applied by imposing the following displacement on $R P_{1}$ : $\left(U_{1}=\left(F_{11}-1\right) l_{0} ; U_{2}=0 ; U_{3}=0\right)$. This reference point is connected with each node pair to apply the MPC represented by Eq. (B.5). The reaction forces induced by the displacement applied on $R P_{1}$ are equal to the components 11, 12 and 13 of the macroscopic first Piola-Kirchhoff stress tensor $\mathbf{P}$ multiplied by the initial surface $\left(l_{0}\right)^{2}$ of face $B_{1}^{-}$(Lejeunes and Bourgeois, 2011; Zhu et al., 2020). Similar developments can be performed to apply the periodic boundary conditions on the other faces.

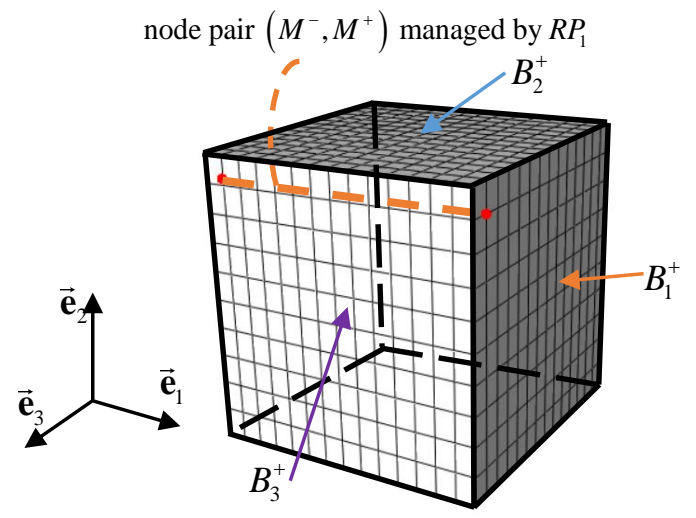

Fig. B. 1. Illustration of the periodic boundary conditions relative to nodes $M^{-}$and $M^{+}$. 


\section{References}

ABAQUS, 2014. User's Manual 6.14, Abaqus Inc.

Akpama, H.K., Ben Bettaieb, M., Abed-Meraim, F., 2017. Localized necking predictions based on rate-independent selfconsistent polycrystal plasticity: Bifurcation analysis versus imperfection approach. Int. J. Plast. 91, $205-237$.

Bao, Y., Wierzbicki, T., 2004. On fracture locus in the equivalent strain and stress triaxiality space. Int. J. Mech. Sci. 46, $81-98$.

Barlat, F., 1987. Crystallographic texture, anisotropic yield surfaces and forming limits of sheet metals. Mater. Sci. Eng. A 91, 55-72.

Barsoum, I., Faleskog, J., 2011. Micromechanical analysis on the influence of the Lode parameter on void growth and coalescence. Int. J. Solids Struct. 48, 925-938.

Barsoum, I., Faleskog, J., 2007. Rupture mechanisms in combined tension and shear-Experiments. Int. J. Solids Struct. 44, 1768-1786.

Ben Bettaieb, M., Abed-Meraim, F., 2015. Investigation of localized necking in substrate-supported metal layers: Comparison of bifurcation and imperfection analyses. Int. J. Plast. 65, 168-190.

Ben Bettaieb, M., Debordes, O., Dogui, A., Duchene, L., 2012. Averaging properties for periodic homogenization and large deformation. Int. J. Multiscale Comput. Eng. 10, 281-293.

Bomarito, G.F., Warner, D.H., 2015. Micromechanical investigation of ductile failure in Al 5083-H116 via 3D unit cell modeling. J. Mech. Phys. Solids 74, 97-110.

Chalal, H., Abed-Meraim, F., 2015. Hardening effects on strain localization predictions in porous ductile materials using the bifurcation approach. Mech. Mater. 91, 152-166.

Dæhli, L.E.B., Morin, D., Børvik, T., Hopperstad, O.S., 2017. Influence of yield surface curvature on the macroscopic yielding and ductile failure of isotropic porous plastic materials. J. Mech. Phys. Solids 107, $253-283$.

Driemeier, L., Brünig, M., Micheli, G., Alves, M., 2010. Experiments on stress-triaxiality dependence of material behavior of aluminum alloys. Mech. Mater. 42, 207-217.

Dunand, M., Mohr, D., 2014. Effect of Lode parameter on plastic flow localization after proportional loading at low stress triaxialities. J. Mech. Phys. Solids 66, 133-153.

Fabrègue, D., Pardoen, T., 2008. A constitutive model for elastoplastic solids containing primary and secondary voids. J. Mech. Phys. Solids 56, 719-741. 
Gao, X., Kim, J., 2006. Modeling of ductile fracture: Significance of void coalescence. Int. J. Solids Struct. 43, 62776293.

Gologanu, M., Leblond, J.B., Devaux, J., 1993. Approximate models for ductile metals containing non-spherical voidscase of axisymmetric prolate ellipsoidal cavities. J. Mech. Phys. Solids 41, 1723-1754.

Gologanu, M., Leblond, J.B., Devaux, J., 1994. Approximate models for ductile metals containing non-spherical voidscase of axisymmetric oblate ellipsoidal cavities. J. Eng. Mater. Technol. 116, 290-297.

Guo, T.F., Wong, W.H., 2018. Void-sheet analysis on macroscopic strain localization and void coalescence. J. Mech. Phys. Solids 118, 172-203.

Gurson, A.L., 1977. Continuum Theory of Ductile Rupture by Void Nucleation and Growth: Part I-Yield Criteria and Flow Rules for Porous Ductile Media. J. Eng. Mater. Technol. 99, 2-15.

Henyš, P., Čapek, L., Březina, J., 2019. Comparison of current methods for implementing periodic boundary conditions in multi-scale homogenization. Eur. J. Mechanics A-Solid, 78, 103825.

Kanit, T., Forest, S., Galliet, I., Mounoury, V., Jeulin, D., 2003. Determination of the size of the representative volume element for random composites: statistical and numerical approach. Int. J. Solids Struct. 40, 3647-3679.

Keralavarma, S.M., Benzerga, A.A., 2010. A constitutive model for plastically anisotropic solids with nonspherical voids. J. Mech. Phys. Solids 58, 874-901.

Koplik, J., Needleman, A., 1988. Void growth and coalescence in porous plastic solids. Int. J. Solids Struct. $24,835-853$.

Lejeunes, S., Bourgeois, S., 2011. Une Toolbox Abaqus pour le calcul de propriétés effectives de milieux hétérogènes, in: 10ème Colloque National En Calcul Des Structures. 1-9.

Liu, Z.G., Wong, W.H., Guo, T.F., 2016. Void behaviors from low to high triaxialities: Transition from void collapse to void coalescence. Int. J. Plast. 84, 183-202.

Luo, T., Gao, X., 2018. On the prediction of ductile fracture by void coalescence and strain localization. J. Mech. Phys. Solids $113,82-104$.

Mansouri, L., Chalal, H., Abed-Meraim, F., 2014. Ductility limit prediction using a GTN damage model coupled with localization bifurcation analysis. Mech. Mater. 76, 64-92.

Marciniak, Z., Kuczynski, K., 1967. Limit strains in processes of stretch-forming sheet metal. Int. J. Mech. Sci. 9, 609620.

Miehe, C., 2003. Computational micro-to-macro transitions for discretized micro-structures of heterogeneous materials at finite strains based on the minimization of averaged incremental energy. Comput. Methods Appl. Mech. Eng. 192, 559-591.

Needleman, A., Tvergaard, V., 1992. Analyses of Plastic Flow Localization in Metals. Appl. Mech. Rev. 45, S3-S18. 
Pardoen, T., Hutchinson, J.W., 2000. An extended model for void growth and coalescence. J. Mech. Phys. Solids 48, $2467-2512$.

Pineau. A., Joly, P., 1991. Local versus global approaches of elastic-plastic fracture mechanics. Application to ferritic steels and a cast duplex stainless steel. Effects Assessment in Components-Fundamentals and Applications (ed. J.C. BLAUEL and K.H. SCHWALBE). ESIS. ECF publication No. 9. Mechanical Engineering Publications Ltd, London.

Rice, J.R., 1976. The localization of plastic deformation. In: Koiter, W.D. (Ed.), Theoretical and Applied Mechanics (Proceedings of the 14th International Congress on Theoretical and Applied Mechanics, Delft), 1. North-Holland Publishing Co., pp. 207-220.

Rudnicki, J.W., Rice, J.R., 1975. Conditions for the localization of deformation in pressure-sensitive dilatant materials. J. Mech. Phys. Solids 23, 371-394.

Swift, H.W., 1952. Plastic instability under plane stress. J. Mech. Phys. Solids 1, 1-18.

Tekoğlu, C., 2015. Void coalescence in ductile solids containing two populations of voids. Eng. Fract. Mech. 147, 418430 .

Tekoğlu, C., Hutchinson, J.W., Pardoen, T., 2015. On localization and void coalescence as a precursor to ductile fracture. Philos. Trans. R. Soc. A Math. Phys. Eng. Sci. 373.

Terada, K., Hori, M., Kyoya, T., Kikuchi, N., 2000. Simulation of the multi-scale convergence in computational homogenization approaches. Int. J. Solids Struct. 37, 2285-2311.

Tvergaard, V., 2012. Effect of stress-state and spacing on voids in a shear-field. Int. J. Solids Struct. 49, 3047-3054.

Tvergaard, V., Needleman, A., 1984. Analysis of the cup-cone fracture in a round tensile bar. Acta Metall. 32, $157-169$.

Wong, W.H., Guo, T.F., 2015. On the energetics of tensile and shear void coalescences. J. Mech. Phys. Solids 82, 259286.

Zhu, J., Ben Bettaieb, M., Abed-Meraim, F., 2020. Numerical investigation of necking in perforated sheets using the periodic homogenization approach. Int. J. Mech. Sci. 166, 105209.

Zhu, Y., Engelhardt, M.D., Kiran, R., 2018. Combined effects of triaxiality, Lode parameter and shear stress on void growth and coalescence. Eng. Fract. Mech. 199, 410-437. 